\title{
Resting-state functional connectivity predicts recovery from visually induced motion sickness
}

\section{AUTHOR(S):}

Miyazaki, Jungo; Yamamoto, Hiroki; Ichimura, Yoshikatsu; Yamashiro, Hiroyuki; Murase, Tomokazu; Yamamoto, Tetsuya; Umeda, Masahiro; Higuchi, Toshihiro

\section{CITATION:}

Miyazaki, Jungo ...[et al]. Resting-state functional connectivity predicts recovery from visually induced motion sickness. Experimental Brain Research 2021, 239: 903-921

\section{ISSUE DATE:}

2021-03

URL:

http://hdl.handle.net/2433/262420

\section{RIGHT:}

This is a post-peer-review, pre-copyedit version of an article published in Experimental Brain Research. The final authenticated version is available online at: http://dx.doi.org/10.1007/s00221-020-06002-7.; The full-text file will be made open to the public on 13 January 2022 in accordance with publisher's 'Terms and Conditions for Self-Archiving'.; This is not the published version. Please cite only the published version. この論文は出版社版でありません。引用の際 には出版社版をご確認ご利用ください。 


\title{
RESEARCH ARTICLES
}

2

3 Resting-state functional connectivity predicts recovery from visually 4 induced motion sickness

5

6

\author{
Jungo Miyazaki ${ }^{1}$, Hiroki Yamamoto ${ }^{2 *}$, Yoshikatsu Ichimura ${ }^{3}$, Hiroyuki \\ Yamashiro $^{4}$, Tomokazu Murase ${ }^{5}$, Tetsuya Yamamoto ${ }^{6}$, Masahiro Umeda ${ }^{7}$, Toshihiro \\ Higuchi $^{5}$
}

\footnotetext{
${ }^{1}$ Research and Development Department, Kyocera Corporation, Kanagawa, Japan ${ }^{2}$ Graduate School of Human and Environmental Studies, Kyoto University, Kyoto,
} Japan

${ }^{3}$ Corporate R\&D, Canon Inc., Tokyo, Japan

${ }^{4}$ Department of Medical Engineering, Aino University, Osaka, Japan ${ }^{5}$ Departments of Neurosurgery, Meiji University of Oriental Medicine, Kyoto, Japan

${ }^{6}$ Department of System Neuroscience, National Institute for Physiological Sciences, Aichi, Japan

${ }^{7}$ Departments of Medical Informatics, Meiji University of Oriental Medicine, Kyoto, Japan

\section{*Correspondence to: Dr. Hiroki Yamamoto}

E-mail: yamamoto@cv.jinkan.kyoto-u.ac.jp

Tel: +81-75-753-2978; Fax: +81-75-753-6574

ORCID: 0000-0003-1051-6599

Keywords: motion sickness, fMRI, recovery, visual cortex, cingulate cortex, insula

\section{Acknowledgements}

2 This study was supported by Grants-in-Aid for Scientific Research on Innovative Areas "Shitsukan" (23135517, 25135720) from the Ministry of Education, Culture, Sports, Science and Technology of Japan and a Grant-in-Aid for Scientific Research (16K13506) from the Japan Society for the Promotion of Science (JSPS) to H. Yamamoto. 
1 Abstract

2 Movies depicting certain types of motion often provoke uncomfortable symptoms similar

3 to motion sickness, termed visually induced motion sickness (VIMS). VIMS generally

4 evolves slowly during the viewing of a motion stimulus and, when the stimulus is

5 removed, the recovery proceeds over time. Recent human neuroimaging studies have

6 provided new insights into the neural bases of the evolution of VIMS. In contrast, no

7 study has investigated the neural correlates of the recovery from VIMS. Study of the

8 recovery process is critical for the development of a way to promote recovery and could

9 provide further clues for understanding the mechanisms of VIMS. We thus investigated

10 brain activity during the recovery from VIMS with functional connectivity magnetic

11 resonance imaging. We found enhanced recovery-related functional connectivity patterns

12 involving brain areas such as the insular, cingulate, and visual cortical regions, which

13 have been suggested to play important roles in the emergence of VIMS. These regions

14 also constituted large interactive networks. Furthermore, the increase in functional

15 connectivity was correlated with the subjective awareness of recovery for the following

16 five pairs of brain regions: insula-superior temporal gyrus, claustrum-left and right

17 inferior parietal lobules, claustrum-superior temporal gyrus, and superior frontal gyrus-

18 lentiform nucleus. Considering the previous findings on the functions of these regions

19 and the present results, it is suggested that the increase in FC may reflect brain processes

20 such as enhanced interoceptive awareness to one's own bodily state, a neuroplastic

21 change in visual processing circuits, and/or the maintenance of visual spatial memory. 


\section{Introduction}

2 First-person perspective images, such as movies captured by action cameras or drones,

3 have recently become popular. Such movies are so realistic that viewers can feel as if they

4 themselves are moving inside the image space. However, the rich image experience is 5 associated with a side effect, termed visually induced motion sickness (VIMS), which 6 involves unpleasant motion sickness (MS)-like symptoms caused by movies depicting 7 certain types of movement. The symptoms are classified into three types: (1) oculomotor 8 (eyestrain, difficulty focusing, headache), (2) nausea (stomach awareness, increased 9 salivation, burping), and (3) disorientation (dizziness, vertigo, drowsiness) (Shupak \& 10 Gordon, 2006; Kennedy et al., 2010). These symptoms can last for more than an hour 11 (Kennedy et al., 1993a; Ujike et al., 2008).

12 VIMS generally emerges and evolves slowly during exposure to a motion stimulus, 13 and, when the stimulus is removed, the individual slowly recovers from the MS symptoms 14 over time. For the emergence and evolutional phase of VIMS, several neuroimaging 15 studies have revealed the underlying brain response (Napadow et al., 2013; Miyazaki et 16 al., 2015; Farmer et al., 2015; Toschi et al., 2017). Napadow et al. (2013) measured the 17 functional magnetic resonance imaging (fMRI) activity of female participants during the 18 presentation of translating black/white stripes for a long enough period to induce VIMS, 19 finding that nausea was associated with a broad network of brain areas including the insula, cingulate cortex, and limbic regions, which are known to process stress, emotion, and interoception. In addition, Miyazaki et al. (2015) measured fMRI visual cortical activities during the evolutional phase of VIMS, which was provoked by the presentation of live-action images containing translational and rotational global motion components. The results showed that the activities of the middle temporal (MT+) area were desynchronised between the left and right hemispheres when participants had MS. The $\mathrm{MT}+$ area has been suggested to interact with the parieto-insular vestibular cortex (PIVC), the centre of vestibular sensation, in the processing of self-motion stimuli that can induce VIMS (Brandt et al., 1998; Kleinschmidt et al., 2002; Smith et al., 2012; Frank et al., 2014, 2016). Taking these results together, the insular, cingulate, visual, vestibular cortical, and limbic regions are likely involved in the evolution of VIMS.

These findings seem to be consistent with several hypotheses concerning the incidence and evolution of MS. The modulation of the MT+ activities could be related to 
1 the eye movement hypothesis (Ebenholtz, 1992; Ebenholtz et al., 1994). This hypothesis

2 proposes that exposure to a specific motion environment could induce abnormal eye

3 movements that would stimulate the MS-related nuclei in the brainstem. The MT+ has

4 been suggested to be intimately involved in eye movement control (Corbetta et al., 1998;

5 Dieterich et al., 1998, 2003; Konen et al., 2005) and therefore might also be associated

6 with the incidence of MS. In addition, the involvement of PIVC may be related to the

7 postural instability theory (Riccio \& Stoffregen, 1991), indicating the importance of

8 postural sway, which would be processed by the PIVC. Additionally, both visual and

9 vestibular signals could be ascribed to the sensory conflict hypothesis (Reason \& Brand,

10 1975; Reason, 1978; Oman, 1990), which proposes that mismatches between multiple

11 sensory inputs such as visual and vestibular signals could lead to MS. Thus, recent

12 neuroimaging studies have provided information on neural correlates of the emergence

13 of VIMS in relation to its existing theories.

14 What happens during recovery from VIMS? Study of the recovery process from 15 VIMS is critical for developing ways to promote recovery from the unpleasant symptoms of VIMS and could also provide clues for further investigation of its mechanisms. The brain processes related to the recovery phase of VIMS are still unclear but previous studies of unpleasant events similar to MS, such as fatigue or stress, may provide some hints. For example, Peltier et al. (2005) reported a fatigue-related reduction in functional connectivity, which was originally defined as a 'temporal correlation between spatially remote neurophysiological events' (Friston et al., 1993), and functional connectivity has since been widely studied to elucidate functionally coordinated behaviour between brain regions. They measured fMRI time-series in the rest phase after participants completed a muscle fatigue task and found decreased functional connectivity between the motor cortices of the left and right hemispheres. In another example, van Marle et al. (2010) measured resting-state fMRI activity soon after female participants experienced psychological stress induced by viewing a strongly aversive movie. They reported increased functional coupling of the amygdala with the dorsal anterior cingulate cortex, anterior insula, and dorsorostral pontine region, indicating the extended state of hypervigilance that promotes sustained salience and mnemonic processing. These examples suggest that, during recovery, brain reorganisation could occur in functional brain networks related to the negative effects. 
We therefore conjectured that such brain reorganisation would also occur during the 2 recovery from VIMS. To test this prediction, we investigated resting-state fMRI activities 3 during the recovery phase of VIMS. Specifically, we predicted that the functional connectivity of brain regions such as the insula, cingulate, visual, and/or vestibular cortical regions would be selectively changed because these regions have been linked to

6 the emergence and evolutional phase of VIMS.

\section{Methods}

9 The present study analysed resting-state data obtained in our previous study (Miyazaki et 10 al., 2015), which provides details of the experiment and the task data.

\section{Participants}

13 Participants were 14 volunteers (12 men and 2 women, including 4 authors; mean age, 1434.9 years; range, 25-48 years) with normal or corrected-to-normal vision. All 15 participants had no history of neurologic or psychiatric disorder and provided written 16 informed consent. All experimental procedures were approved by the Human Studies

17 Committee of the Graduate School of Human and Environmental Studies at Kyoto 18 University and the Department of Neurosurgery at Meiji University of Oriental Medicine. 19 Participants were classified into two groups: the VIMS group $(n=6$, all men including 202 authors; mean age \pm SD, $34.7 \pm 8.0$ years) who experienced VIMS in the experimental 21 session; and the healthy group $(\mathrm{n}=6,2$ women and 4 men including 2 authors; mean age $22 \pm \mathrm{SD}, 33.3 \pm 7.2$ years) who did not experience VIMS. A possible concern is that the 23 recruitment of some authors as participants would affect our results. We thus conducted 24 three types of statistical analyses to address this possibility: a sub-analysis excluding the 25 author participants, an analysis calculating the interclass correlation and design effect of authorship, and a linear mixed-effects model using authorship as one of the factors. None of the analyses suggested substantial effects of authorship.

28 The VIMS or healthy classification was performed using a self-reported VIMS (yes or no) by asking, 'Did you get motion sick during the experimental session?'. Two of the 30 participants in the VIMS group were subsequently excluded from the analysis of the 31 recovery process because they had not recovered from the VIMS according to the 32 Simulator Sickness Questionnaire (SSQ) score (Kennedy et al., 1993b). Briefly, the SSQ 
1 was developed as a standard measure for evaluating the severity of simulator sickness and

2 has also been used to measure VIMS symptoms. However, several studies (Stanney et al., 3 1998; Stoffregen et al., 2019; Walter et al., 2019) have indicated that the SSQ could

4 deviate from self-reports. Such a discrepancy was not observed in the present study. The

5 SSQ scores of the VIMS group markedly increased immediately after the VIMS-inducing

6 stimulus and decreased to a normal level during the subsequent rest (recovery) phase

7 (except for the abovementioned 2 participants), whereas the SSQ scores of the healthy 8 group did not change throughout the experimental session (see Figure 4 in Miyazaki et $9 a l$. [2015]), suggesting that the SSQ scores show a similar trend to the self-reports.

\section{Visual stimuli}

12 In the experiment, two types of visual stimuli-a global motion stimulus and a local 13 one-were presented. The global motion stimulus was a 6-min-long live-action movie 14 containing first-person-perspective, global visual motion. This stimulus content could 15 induce VIMS in viewers. In contrast, the local motion stimulus was a 6-min-long liveaction movie consisting of 8 by 8 patches (total, 64), which reduced the global motion stimulus to one-eighth vertically and horizontally. This stimulus was a control and did not induce VIMS in viewers because the stimulus contained no global motion component that could trigger VIMS. The stimulus was based on Wall and Smith (2008).

These stimuli were projected onto a screen located over the participant's forehead using a DLP projector (LVP-HC3800; Mitsubishi, Japan). The spatial resolution was 1080 $\times 720$ pixels, and the refresh rate was $60 \mathrm{~Hz}$. The participant was supine and viewed the stimuli through a planar mirror located $25 \mathrm{~cm}$ from the eyes. See Miyazaki et al. (2015) 24 for details.

\section{Experimental procedure}

27 The experimental procedure is shown in Figure 1. Before and after the presentation of the 28 two aforementioned stimuli, three rest phases (Rest-1, Rest-2, and Rest-3) were arranged. 29 Each rest phase was 5 min long and comprised presentation of a grey background with a 30 fixation point at the centre. Before the experimental session, participants were instructed 31 to relax with their eyes open during the rest phases, and participants were allowed to leave 32 the experiment at any time if they could not continue the session. Throughout the session, 
1 participants lay supine on the MRI scanner table without firm restriction of their head or 2 body so as to minimise undesired effects such as pain and distraction. After the first rest 3 phase (Rest-1), participants viewed the local motion stimulus; none experienced VIMS. 4 Next, the participants experienced the second rest phase (Rest-2) and then viewed the 5 global motion stimulus; 8 of the 14 participants experienced VIMS, as described above. 6 The third rest phase (Rest-3) followed. The order of the stimulus presentations (the local 7 motion stimulus followed by the global one) was not balanced to avoid a carryover effect 8 from the global to the local motion period because our preliminary experiment had 9 indicated that, when the global stimulus precedes the local one, the VIMS symptoms can 10 be prolonged and overlap the subsequent local stimulus presentation.

11 To rate the degree of VIMS symptoms, participants answered the Simulator Sickness 12 Questionnaire (SSQ) (Kennedy et al., 1993b) 6 times in total, that is, before and after 13 each stimulus presentation and each rest phase. The SSQ was developed as a metric of 14 simulator sickness, and this metric consists of 16 questions on symptoms such as 15 headache and nausea, based on four grades. The SSQ has generally also been used as a metric for VIMS. The question items of the SSQ were presented to participants through images, and the questions were answered by use of a response pad with four buttons (HH1x4L; Current Design Inc., Philadelphia, PA). A decrease in the SSQ scores between

19 before and after the rest phases, which would reflect recovery from VIMS, was analysed 20 because the aim of the present study was to investigate the recovery phase of VIMS at 21 rest.

(Please place Figure 1 around here)

\section{MRI data acquisition}

26 Functional MR images were acquired using a clinical 3-T MR scanner (Trio TIM; 27 Siemens, Germany) and a 20-channel phased-array head coil with a $\mathrm{T}^{*}$-weighted 28 gradient-echo echo planar sequence (repetition time $[\mathrm{TR}]=2000 \mathrm{~ms}$; echo time $[\mathrm{TE}]=$ $2930 \mathrm{~ms}$; flip angle $[\mathrm{FA}]=90^{\circ}$; voxel size $[\mathrm{VS}]=3 \mathrm{~mm} \times 3 \mathrm{~mm} \times 4 \mathrm{~mm}$; field of view $30[\mathrm{FOV}]=192 \mathrm{~mm} \times 192 \mathrm{~mm} ; 37$ slices; axial orientation parallel to the AC-PC plane; 31 interleaved acquisition). The functional images were continuously scanned from $10 \mathrm{~s}$ before Rest- 1 to the end of Rest-3, taking about $35 \mathrm{~min}$. For anatomical registration, brain 
1 structural images were acquired using a 3D magnetisation-prepared rapid gradient-echo

2 T1-weighted sequence with the following parameters: $\mathrm{TR}=1800 \mathrm{~ms}$, TE $=3.03 \mathrm{~ms}$,

3 inversion time $=650 \mathrm{~ms}, \mathrm{FA}=9^{\circ}, \mathrm{VS}=0.8 \mathrm{~mm} \times 0.8 \mathrm{~mm} \times 0.8 \mathrm{~mm}, \mathrm{FOV}=205 \mathrm{~mm} \times$

$4205 \mathrm{~mm}, 176$ slices, axial orientation parallel to the AC-PC plane, interleaved acquisition. 5

6 Behavioural data analyses

$7 \quad$ Visually induced motion sickness

8 After the experimental session, participants self-reported whether or not they experienced

9 VIMS while viewing the global motion stimulus and were provisionally classified into 10 the VIMS or healthy group based on the self-report as mentioned above. To objectively 11 test the validity of this grouping criterion based on the self-reports, SSQ scores, which 12 are a metric for MS symptoms, were statistically tested to determine whether the scores 13 of the VIMS group selectively increased after the global motion stimulus. Details of this 14 analysis are provided in Miyazaki et al. (2015).

Recovery from visually induced motion sickness

17 Next, to verify that the VIMS group participants recovered during Rest-3, the differences in the SSQ scores between before and after each rest phase were computed, and the differences were statistically tested to determine whether the score of the VIMS group became significantly larger only for Rest-3, reflecting the recovery from VIMS, and whether the scores of the other rest phases did not change. To facilitate interpretation of the results, we examined not only the SSQ Total Score, but also the other three subscales: Oculomotor, Nausea, and Disorientation. This analysis was performed using R software (R Core Team, 2017) (see also Miyazaki et al., 2015). A linear mixed-effects model analysis was performed with the lmer function of the lme4 package (Bates et al., 2014). This model had a within-participants fixed effect PHASE (Rest-1 vs. Rest-2 vs. Rest-3), a between-participants fixed effect GROUP (VIMS vs. healthy participant groups), an interaction of PHASE and GROUP, and a random effect of each individual participant. This analysis was conducted separately for the four scales of the SSQ (Total Score, Nausea, Oculomotor, and Disorientation).

When the PHASE $\times$ GROUP interaction was statistically significant, for the post hoc analysis, the simple effect of PHASE was tested separately for the VIMS and healthy 
1 groups. Specifically, the differences in scores between the levels of PHASE (i.e., three 2 ways of Rest-1 vs. Rest-2; Rest-2 vs. Rest-3; and Rest-1 vs. Rest-3) were tested by means 3 of the functions testInteractions and testFactors of the phia package (De Rosario4 Martinez, 2015) separately for each participant's group. In contrast, when the interaction 5 was not significant, the main effect of PHASE was reported instead. The significance 6 level for all statistical analyses was set to 0.05 , with correction by the Bonferroni method 7 for multiple comparisons involving the four scales of the SSQ, the two participant groups, and the repetition of the post hoc tests.

\section{0 fMRI data analyses}

\section{Preprocessing}

12 All fMRI data were preprocessed using AFNI software (Cox, 1996) as follows: (1) slice 13 timing correction; (2) head motion correction; (3) extraction of 5-min-long time-series 14 signals (150 fMRI volumes) acquired during each rest phase; (4) transformation to the 15 standard Talairach space for group analysis; (5) smoothing with the Gaussian kernel of an isotropic 9-mm FWHM; (6) band-pass filtering with a cutoff of 0.01-0.1 Hz; and (7) removal of fMRI volumes whose motion or its derivatives exceeded $0.2 \mathrm{~mm}$ (i.e., motion 'scrubbing') to reduce the effect of head movement on fMRI data, in addition to the removal of outlier volumes where the ratio of the number of outlier voxels exceeded 0.1. 20

\section{Brain connectedness mapping}

After the preprocessing, connectedness maps were made by use of the AFNI 3dTcorrMap function. Connectedness is the average of the Pearson correlation coefficients between a specific voxel time-series and the other in the brain mask (Gotts et al., 2012). The Pearson correlation coefficient is a real number ranging from -1 to +1 , and this value was converted to a Fisher's Z value with the 3dTCorrMap function for the following analyses. The $\mathrm{Z}$ values were compared between the VIMS and healthy groups within the Talairach space by using the AFNI $3 d L M E$ function. For the mapping of the results of this comparison, the voxel-wise $P$ value was set to 0.0001 . The false-positive rate for the clustering was estimated by use of the $3 d$ ClustSim function, in which the threshold $\alpha$ for the cluster size was set to 0.10 ; this relatively loose threshold was used because this was a first screening procedure to restrict candidates. The conventional 0.05 threshold was 
1 used in the second screening based on functional connectivity described below. Based on

2 this mapping, the regions whose connectedness changed significantly during the recovery

3 phase of VIMS were detected. Specifically, the regions were determined according to the

4 following criteria: the connectedness was changed only for the VIMS group selectively

5 during Rest-3 when the VIMS participants experienced VIMS, whereas that of the healthy

6 group did not change for all rest phases (interaction contrast calculated using the $3 d L M E$

7 function: absolute intergroup difference in $\mathrm{Z}_{\text {Rest-3 }}-0.5 \times \mathrm{Z}_{\text {Rest-1 }}-0.5 \times \mathrm{Z}_{\text {Rest-2: }}$ : the VIMS

8 group - the healthy group $>3.891$, which corresponds to $P<0.0001$ ).

9 Through these steps, 12 brain regions were identified with significant changes in

10 connectedness during recovery from VIMS (described below). In each region, the mean

11 time-series averaged across voxels within the region was calculated and used as a seed

12 for the functional connectivity analysis in the following section.

\section{ROI-based functional connectivity analysis}

15 As stated above, the mean time-series averaged across voxels within the brain regions showing significant changes in connectedness during recovery from VIMS were used as seeds to examine functional connectivity throughout the entire brain. Then, using the same method as in the analysis of connectedness, functional connectivity intensity was compared between the VIMS and healthy groups. After adjustment to the false-positive rate for clustering, the result was mapped in Talairach space (voxel-wise $P$ value $=0.0001$, $\alpha=0.05)$, through which the brain regions with changes in functional connectivity during recovery from VIMS that fulfilled the following criteria were identified: (i) functional connectivity in Rest-3 following the onset of VIMS changed only in the VIMS group (interaction contrast calculated using the $3 d L M E$ function: absolute intergroup difference in $Z_{\text {Rest-3 }}-0.5 \times Z_{\text {Rest-1 }}-0.5 \times Z_{\text {Rest-2: }}$ the VIMS group-the healthy group $>3.891$, which corresponds to $P<0.0001$ ) and (ii) neither group had a change in functional connectivity during other rest phases (the VIMS group, $\left|Z_{\text {Rest-2 }}-Z_{\text {Rest-1 }}\right|<1.96$, which corresponds to $28 P>0.05$; the healthy group, $\left|\mathrm{Z}_{\text {Rest-3 }}-0.5 \times \mathrm{Z}_{\text {Rest-1 }}-0.5 \times \mathrm{Z}_{\text {Rest-2 }}\right|<1.96 ;\left|\mathrm{Z}_{\text {Rest-2 }}-\mathrm{Z}_{\text {Rest-1 }}\right|$ $<1.96)$.

This functional connectivity analysis extracted 19 pairs of brain regions, and changes in each functional connectivity were analysed using R software (R Core Team, 2017) and 
1 consisting of a within-participants fixed effect (PHASE; Rest-1 vs. Rest-2 vs. Rest-3), a 2 between-participants fixed effect (GROUP; VIMS vs. the healthy group), an interaction 3 of PHASE and GROUP, and a random effect of each individual participant.

Because the PHASE $\times$ GROUP interactions for all region pairs were statistically significant, a post hoc test was performed to analyse the simple main effect of PHASE in the VIMS and healthy groups separately. Specifically, the differences in connectivity between Rest- 1 and Rest-2, Rest-2 and Rest-3, and Rest-1 and Rest- 3 were tested in each group. The Bonferroni method was used to correct multiple comparisons for functional connectivities in the 19 pairs of brain regions, the two participant groups, and repeated post hoc tests of the three PHASE combinations.

\section{Correlation analysis}

Correlation analysis was performed to clarify whether a change in the SSQ Total Score (i.e., subjective awareness of recovery) could be predicted from the change in functional connectivity. Because exceptionally large changes in SSQ Total Scores were observed in some participants, the Spearman's rank correlation coefficient, a nonparametric statistic that is seldom affected by such variation, was used to analyse the 19 pairs of brain regions separately. Because of the multiple comparisons, $P$ values were subjected to Bonferroni correction.

\section{Global network analyses}

2 To analyse the functional network of the brain regions showing recovery-related increases 3 in connectedness, a multivariate analysis was performed. First, for each participant and 24 for each rest phase, a partial correlation coefficient of the fMRI time-series was computed 5 for each pair of the 12 brain regions, resulting in a $12 \times 12$ matrix. The partial correlation 6 matrix was then converted into a distance matrix, where the distance was defined by 1 7 minus the absolute partial correlation coefficient. Second, for each rest phase, within8 group averaged distance matrices were computed for VIMS and healthy groups. The 29 distance matrices of the first phase (Rest-1) and second phase (Rest-2) were further 30 averaged as a control for the recovery phase (Rest-3). Third, the columns and rows of the 1 distance matrices were reordered to place similar brain regions closer, using the seriation 2 function with the 'HC' option in the seriation package (Hahsler et al., 2008) of R software. 
1 Finally, dendrograms, which are hierarchical clustering trees, were derived from the

2 distance matrices using the pvclust package (Suzuki \& Shimodaira, 2019) of R software,

3 which provided $P$ values of each cluster through multiscale bootstrap resampling.

4 Independently of the above analyses, statistically independent brain networks were

5 extracted by a dictionary learning framework. The procedure and the results are detailed

6 in the Supplementary Materials.

\section{Results}

9 Behavioural results

10 Details of the results for VIMS symptoms induced in the experiment are provided in 11 Miyazaki et al. (2015). In brief, 8 of the 14 participants experienced VIMS due to the 12 global motion stimulus, and the remaining 6 did not (Figs. 3 and 4 of Miyazaki et al. 13 [2015]). These results were confirmed by statistical analysis of the SSQ scores (Table 1 14 of Miyazaki et al. [2015]). Participants were divided into VIMS $(\mathrm{n}=8)$ and healthy $(\mathrm{n}=$ 15 6) groups based on the presence and absence of VIMS, respectively. However, 2 of the 8 16 participants in the VIMS group did not recover from the VIMS even after the 17 experimental session, and this was reflected in their SSQ scores, which did not decrease even after Rest-3. Because the aim of this study was to analyse the recovery phase of VIMS, these 2 participants were excluded from the subsequent analyses.

To verify that the participants in the VIMS group had recovered from VIMS during Rest-3, a linear mixed-effects model analysis of the SSQ score was performed before and after each rest phase (Table 1 and Fig. 2). SSQ Total Scores decreased during Rest-3 only in the VIMS group. The interaction of PHASE and GROUP was statistically significant $\left(\chi^{2}(2)=13.30, P=0.005\right.$, Bonferroni corrected). A post hoc test of the simple main effect of PHASE showed that SSQ scores decreased significantly during Rest-3 only in the VIMS group (the VIMS group: Rest- 1 vs. Rest-2: $\chi^{2}(2)=0.03, P>1$; Rest-2 vs. Rest-3: $\chi^{2}(2)=15.04, P=0.001$; Rest-1 vs. Rest-3: $\chi^{2}(2)=16.41, P=0.0007$; the healthy group: Rest-1 vs. Rest-2: $\chi^{2}(2)=4.72, P=0.417$; Rest-2 vs. Rest-3: $\chi^{2}(2)=0.07, P>1$; Rest-1 vs. Rest- $3: \chi^{2}(2)=5.98, P=0.203$, Bonferroni corrected), verifying that the participants in the VIMS group recovered from the VIMS during Rest-3.

Among the three SSQ subscales, the Disorientation score was similar to the Total Score (PHASE $\times$ GROUP interaction: $\chi^{2}(2)=19.16, P=0.0002$; post hoc test: the VIMS 
1 group: Rest-1 vs. Rest-2: $\chi^{2}(2)=0.02, P>1$; Rest-2 vs. Rest-3: $\chi^{2}(2)=18.52, P=0.0002$;

2 Rest-1 vs. Rest-3: $\chi^{2}(2)=19.87, P=0.0001$; the healthy group: Rest-1 vs. Rest-2: $\chi^{2}(2)=$ $30.53, P>1$; Rest-2 vs. Rest-3: $\chi^{2}(2)<0.01, P>1$; Rest-1 vs. Rest-3: $\chi^{2}(2)=0.52, P>1$,

4 Bonferroni corrected). As for Nausea and Oculomotor, the PHASE $\times$ GROUP interactions 5 were not significant (Nausea, $\chi^{2}(2)=7.74, P=0.084$; Oculomotor, $\chi^{2}(2)=5.48, P=$ 6 0.259; Bonferroni corrected), and the main effect of PHASE was also not significant 7 (Nausea, $\chi^{2}(2)=4.28, P=0.539$; Oculomotor, $\chi^{2}(2)=4.62, P=0.444$; Bonferroni 8 corrected).

10 (Please place Figure 2 and Table 1 around here)

\section{Brain connectedness mapping}

13 To test our hypothesis that the recovery phase of VIMS induces changes in functional 14 connectivity in some brain regions, we examined the substantial changes in functional 15

(Please place Figure 3 and Table 2 around here)

\section{ROI-based functional connectivity analyses} connectivity by calculating connectedness, which is the mean correlation coefficient of a specific voxel time-series to other voxel time-series (Gotts et al., 2012), and then by mapping the results in Talairach space. As anticipated, the map revealed 12 brain regions with significant increases in connectedness during Rest-3 in the VIMS group (Table 2 and Fig. 3). The 12 regions included the primary visual cortex in the occipital cortex (the left and right lingual gyrus; Fig. 3k and g, respectively) and the left and right insula (Fig. 3c and d) and cingulate regions (the right anterior cingulate and left cingulate gyrus; Fig. 3b and $\mathrm{h}$, respectively). In addition, the left superior frontal gyrus (Fig. 3j) and the right inferior frontal gyrus (Fig. 31) were included in the 12 brain regions. Furthermore, connectedness significantly increased in the cerebellum (left cerebellar tonsil; Fig. 3e) and the subcortical region (left and right thalamus and the right claustrum; Fig. 3a, i, and f, respectively) during the recovery phase of VIMS. In contrast, no brain region had a significant decrease in connectedness during the recovery phase.

For the 12 ROIs with increased connectedness, the question arose as to whether functional 
1 connectivity had increased in a broad area or in only specific regions. To address this

2 question, the entire brain was screened for functional connectivity using the 12 ROIs as

3 seeds. It was found that 8 of the 12 seed regions showed increases in functional connectivity with specific regions during only Rest-3 (recovery phase) for the VIMS group. These regions were the left thalamus, left and right insula, left cerebellar tonsil,

6 right claustrum, left cingulate gyrus, left superior frontal gyrus, and left inferior frontal

7 gyrus. Each of these regions had selective increases in functional connectivity with 8 several other regions, resulting in the following 19 region pairs: left thalamus-right insula, 9 left thalamus-right inferior parietal lobule, left thalamus-right declive, left insula-left thalamus, left insula-left inferior parietal lobule, right insula-left superior temporal gyrus,

11 right insula-left inferior temporal gyrus, left cerebellar tonsil-left parahippocampal gyrus,

12 right claustrum-left cingulate gyrus, right claustrum-right inferior parietal lobule, right

13 claustrum-left inferior frontal gyrus, right claustrum-left superior temporal gyrus, right

14 claustrum-left inferior parietal lobule, left cingulate gyrus-right insula, left superior 15 frontal gyrus-left medial frontal gyrus, left superior frontal gyrus-left middle temporal gyrus, left superior frontal gyrus-left lentiform nucleus, and right inferior frontal gyrusleft inferior frontal gyrus (Table 3). The locations of the 19 pairs are shown in the left panels of Figure 4 and Supplementary Figure 1. The other 4 of the 12 seed regions had no selective changes in functional connectivity.

The phase (Rest-1, -2 , and -3 ) $\times$ group (Healthy and VIMS) interaction effect can be clearly seen in the right panels of Figure 4 and Supplementary Figure 1. The functional connectivity of the VIMS group (yellow solid line) remained comparable in Rest-1 and Rest-2 but increased in Rest-3 (recovery phase), whereas that of the healthy group (white broken line) did not show an increase in any phase. These results were statistically confirmed by a linear mixed-effects model analysis. The interaction effects of resting phases (PHASE: Rest-1, Rest-2, and Rest-3) and participants' group (GROUP: VIMS and healthy) were statistically significant for all 19 pairs (Table 3). We thus analysed the simple effect of PHASE separately for the VIMS and healthy groups. The statistical results for the VIMS and healthy groups are shown in Tables 4 and 5, respectively. The selective increases in functional connectivity for the VIMS group during Rest-3 (recovery phase) were statistically significant for almost all pairs and the effect sizes (Cohen's $d$ ) were relatively large (Table 4). For example, for the right insula-left thalamus pair (\#6 in 
1 Tables 4 and 5), the simple effect of the Rest-3 PHASE was statistically significant for 2 only the VIMS group (Rest-2 vs. Rest-3: $\chi^{2}(2)=53.99, P=2 \times 10^{-11}$, Cohen's $d=3.00$;

3 Rest-1 vs. Rest-3: $\chi^{2}(2)=38.05, P=8 \times 10^{-8}$, Cohen's $d=2.52$, Bonferroni corrected)

4 (\#6 in Table 4), whereas this effect was not found for the other control conditions (VIMS 5 group: Rest-1 vs. Rest-2: $\chi^{2}(2)=1.39, P>1$, Cohen's $d=0.48$; healthy group: Rest-1 vs. 6 Rest-2: $\chi^{2}(2)=0.13, P>1$, Cohen's $d=0.15$; Rest-2 vs. Rest-3: $\chi^{2}(2)=0.88, P>1$, 7 Cohen's $d=0.38$; Rest-1 vs. Rest-3: $\chi^{2}(2)=1.70, P>1$, Cohen's $d=0.53$, Bonferroni corrected) (\#6 in Tables 4 and 5). Similar selective changes in functional connectivity were observed in the other 18 region pairs (see Tables 3, 4, and 5). However, as an exception, the functional connectivity between the left superior frontal gyrus and left middle temporal gyrus showed a tendency toward an increase in the second $v s$. third rest 13

\section{Correlation analyses}

17 To determine whether the increases in functional connectivity were associated with subjective recovery from VIMS, we conducted correlation analyses between the change in functional connectivity and that in the SSQ Total Scores for the 19 pairs of brain regions

(Fig. 5). The following 5 pairs showed statistically significant negative correlations, indicating regions in which greater increases in functional connectivity were associated with greater recovery from VIMS: the right insula-left superior temporal gyrus (Spearman's rank correlation $\rho=-0.83, P=0.017$, Fig. 5a), right claustrum-right inferior parietal lobule $(\rho=-0.83, P=0.016$, Fig. $5 b$ ), right claustrum-left superior temporal gyrus $(\rho=-0.92, P=0.0004$, Fig. 5c), right claustrum-left inferior parietal lobule $(\rho=$ $-0.80, P=0.038$, Fig. 5d), and left superior frontal gyrus-left lentiform nucleus ( $\rho=$ $-0.80, P=0.038$, Fig. 5e; all Bonferroni corrected). The correlation was not statistically significant in the remaining 14 pairs: the left thalamus-right insula $(\rho=-0.64, P=0.469)$, left thalamus-right inferior parietal lobule $(\rho=-0.63, P=0.510)$, left thalamus-right declive $(\rho=-0.71, P=0.181)$, left insula-left thalamus $(\rho=-0.73, P=0.137)$, left insulaleft inferior parietal lobule ( $\rho=-0.62, P=0.576)$, right insula-left thalamus $(\rho=-0.67$, $P=0.330)$, right insula-left inferior temporal gyrus $(\rho=-0.61, P=0.647)$, left cerebellar 
1 tonsil-left parahippocampal gyrus ( $\rho=-0.71, P=0.181$ ), right claustrum-left cingulate 2 gyrus ( $\rho=-0.75, P=0.095)$, right claustrum-left inferior frontal gyrus $(\rho=-0.74, P=$

$30.115)$, left cingulate gyrus-right insula $(\rho=-0.70, P=0.212$ ), left superior frontal gyrus-

4 left medial frontal gyrus ( $\rho=-0.63, P=0.510$ ), left superior frontal gyrus-left middle temporal gyrus ( $\rho=-0.58, P=0.932$ ), and right inferior frontal gyrus-left inferior frontal gyrus $(\rho=-0.61, P=0.698$; all Bonferroni corrected $)$.

$8 \quad$ (Please place Figure 5 around here)

\section{Global network analyses}

11 The above connectedness map shows the 12 brain regions with recovery-selective increases in connectedness (Fig. 3 and Table 2). To explore the possibility that these regions constituted larger functional networks, we conducted a multivariate analysis in which the network of these regions was analysed based on distance matrices representing dissimilarity among the fMRI time-series in each pair of these regions (Supplementary Figure 2), from which hierarchical clustering dendrograms were derived. Figure 6 compares the results of the VIMS group (a, c) with those of the healthy group (b, d). As clearly shown in Fig. 6, the 12 brain regions of the VIMS group showed more global network structures for both recovery (Rest-3) and non-recovery (the average of Rest-1 and Rest-2) phases, in contrast to the healthy group, for which there was only a statistically significant cluster (marked with asterisks) of the left and right lingual gyri. comprised the brain regions with recovery-selective increases in ROI-based functional connectivity (marked with underlines) and the regions with correlation with the SSQ (marked with italics), corroborating the above analyses (Figs. 4 and 5). During the recovery phase (Rest-3), the left thalamus additionally participated in the visual cortical cluster, and this cluster was combined with the other cluster of the left/right insular and cingulate areas in a higher level. Interestingly, during the phases before sickness developed, the VIMS group already showed a distinct cluster composed of the left cingulate gyrus and right thalamus in conjunction with the visual cortical cluster. Some of these networks were also independently confirmed by dictionary learning-based 
1 connectivity mapping (Supplementary Figure 3).

2

3 (Please place Figure 6 around here)

\section{Discussion}

6 Here, we investigated resting-state fMRI functional connectivity during the recovery

7 phase of VIMS. The analysis showed increased functional connectivity in some brain

8 areas, including the insula, cingulate, and visual cortical regions. These regions were also

9 found to constitute large interactive networks. Furthermore, some of the connectivity 10 increases were strongly correlated with the subjective awareness of recovery from VIMS 11 (decrease in subjective SSQ scores) for the following five pairs of brain regions: the 12 insula-superior temporal gyrus, claustrum-left inferior parietal lobule, claustrum-right 13 inferior parietal lobule, claustrum-superior temporal gyrus, and superior frontal gyrus14 lentiform nucleus. These brain regions included key areas related to the emergence and evolution of VIMS. The insula had increased connectivity with the cingulate gyrus during 16 recovery from VIMS (Supplementary Figure 1k). The insular and cingulate regions have 17 also exhibited a correlated activation during a higher nausea state (Napadow et al., 2013). 18 Additionally, the insula showed a significant correlation between sympathovagal balance 19 and connectivity with a middle temporal region (MT+/V5) during a visually induced nausea sensation (Toschi et al., 2017), which is a visual motion-sensitive area. Moreover, the middle temporal region has exhibited decreased inter-hemispheric synchronisation during VIMS (Miyazaki et al., 2015). Here, this key area had increased connectivity with the superior frontal region during recovery from VIMS (Supplementary Figure 1m). In addition to these key brain areas, several regions detected in our analysis of the recovery from VIMS, such as the inferior frontal gyrus (Supplementary Figure $1 \mathrm{j}$ and $1 \mathrm{n}$ ), cerebellar tonsil (Supplementary Figure 1h), and declive (Supplementary Figure 1c), have been linked to the evolution of VIMS (Farmer et al., 2015). These results thus conformed with our initial expectations that key brain regions related to the emergence and evolution of VIMS could also be involved in the recovery. Further studies are required to verify this speculation because the present work is a correlational study that cannot establish a causal relationship.

In our experiment, the 2 female participants did not experience motion sick and were 
1 included in the healthy group. This result is somewhat inconsistent with the view that 2 women are more susceptible to MS than men (Munafo et al., 2017). This may be simply 3 due to the small sample size and/or the difference in experimental stimuli. The type of visual motion stimuli has been reported to affect the sex difference in MS incidence: the translational motion stimulus induced a sex difference in MS incidence, whereas the

6 rotational one did not (Klosterhalfen et al., 2006; Koslucher et al., 2015; Munafo et al., 7 2017). It thus seems that our global motion stimulus, which included a rotational component caused by camera rotation, might have difficulty inducing a sex difference.

A closer look at the changes in functional connectivity along the resting phases (Rest1, Rest-2, and Rest-3) (Fig. 4) suggests two different groups that might have distinct roles among the five pairs of brain regions. For the first group, the right insula-superior temporal gyrus (Fig. 4a) and the superior frontal gyrus-lentiform nucleus (Fig. 4e), functional connectivity remained roughly comparable in the healthy and VIMS groups before the VIMS (Rest-1 and Rest-2) but increased only in the VIMS group during the recovery from VIMS (Rest-3), indicating temporary reorganisations of neural circuitry triggered by the experience of unusual motion environments. In contrast, for the second group, the claustrum-inferior parietal lobule (Fig. 4b and 4d), functional connectivity was weaker in the VIMS group than in the healthy group before the onset of VIMS but became comparable in the two groups during the recovery from VIMS. A similar intrinsic difference between the groups was also observed in the dendrogram (Fig. 6c vs. Fig. 6d). These results imply that VIMS group participants might have weaker connectivities between specific brain regions, possibly reflecting VMIS susceptibility and/or tolerance. It would be interesting to address MS susceptibility and/or tolerance in a future study with a larger sample size.

The main limitations of this study include its small sample size and its correlational nature. However, this is the first study to elucidate the brain process in the recovery phase of VIMS. Thus, it would be interesting to discuss possible reasons why the functional connectivity between the brain regions increased during the recovery from VIMS. Here, we propose three possibilities and discuss them in relation to the current understanding of VIMS and related hypotheses. The first possibility is that the changes in functional connectivity observed during the recovery phase could be related to interoceptive awareness (perception of one's own bodily state) because interoceptive awareness is 
1 expected to increase to alleviate the symptoms of VIMS, such as dizziness, eye pain, and 2 nausea. In this regard, it is interesting that connectivities, including those of insular and 3 cingulate regions, were increased in our study. The insular cortex, the likely centre of the 4 neural basis of interoception, is also regarded as the limbic sensory cortex (Craig, 2002; 5 Craig \& Craig, 2009) because this brain site might be involved in the homeostatic 6 regulation of the brain stem. In addition, the cingulate gyrus is also known as the limbic 7 motor cortex and might contribute to interoception together with the insular cortex (Craig, 8 2002; Craig \& Craig, 2009). Indeed, according to Napadow et al. (2013), the cingulate 9 cortex is coactivated with the insular cortex when the perception of nausea is triggered by visual stimuli. The claustrum, which had functional connectivity with multiple brain 11 regions in this study, has been reported to be involved in the processing of conscious 12 perception, suggesting its association with interoceptive perception (Crick \& Koch, 2005). 13 Therefore, the strengthened brain networks centring on the insula and claustrum in this 14 study might reflect the internal perception of a poor physical state triggered by VIMS. 15 Similarly, after observing the strengthening of the amygdala network (including the insular cortex) immediately after the induction of psychological stress by visual stimuli, van Marle et al. (2009) suggested that the strengthened amygdala network might reflect negative hypervigilance.

The second possibility is that the changes in functional connectivity observed during the recovery phase of VIMS could reflect plastic changes in neural circuits related to visual processing, such as visual attention and eye movement control. In this regard, we note that visual processing regions, including the middle temporal gyrus, which is a visual motion-sensitive area, exhibited significantly increased functional connectivity during the recovery phase of VIMS in our study. Indeed, the middle temporal gyrus had strong connectivity with the superior frontal gyrus (Supplementary Figure 1m and \#17 in Tables 3,4 , and 5). Interestingly, similar results were reported in previous neuroimaging studies of visual or motor learning (Albert et al., 2009a, b; Lewis et al., 2009; Stevens et al., 2009, 2015). Stevens et al. (2009) showed that functional connectivities between the neural network in the occipital face and scene areas and frontal cortex were increased during the rest phase immediately after a visual face and scene recognition task and that subsequent memory retrieval performance was predicted by the extent of the changes in the functional connectivity. This suggests that a functional connectivity increase during a 
1 rest phase after a task might reflect the neuroplastic change needed to improve the efficacy

2 of task processing. In addition to this change in connectivity between occipital visual and

3 frontal regions, brain areas showing increased functional connectivity in this study (the

4 insula-superior temporal gyrus [Figure 4A], insula-inferior temporal gyrus

5 [Supplementary Figure 1g], cingulate gyrus-insula [Supplementary Figure 1k], superior

6 frontal gyrus-medial frontal gyrus [Supplementary Figure 11], and left and right inferior

7 frontal gyrus [Supplementary Figure 1n]) were coactivated when regulating visual 8 attention or eye movement in previous fMRI studies (Corbetta et al., 1998; Dieterich et 9 al., 1998, 2003; Konen et al., 2005). As for the increased connectivity of the claustrum to 10 multiple brain regions in this study, this area is related to visual and multisensory attention 11 and was reported to be activated at the same time as the inferior parietal lobule and interior 12 frontal gyrus (Vohn et al., 2007), both of which were detected in this study. Attention is 13 closely associated with eye movement (Corbetta et al., 1998), and eye movement has 14 been proposed to cause MS, including VIMS, in the eye movement hypothesis 15 (Ebenholtz, 1992; Ebenholtz et al., 1994). The eye movement hypothesis posits that MS is caused by abnormal eye movement induced in a certain type of motion environment. Given this prior knowledge and our results, the increase in functional connectivity observed during the recovery from VIMS might reflect a plastic change in neural circuits related to visual processing, such as visual attention and/or eye movement control.

Another putative neuronal circuit of VIMS is the vestibular cortex, which is concerned with balance sensation. Previous neuroimaging studies employing visual and vestibular stimuli suggested a close cooperation between the motion-sensitive visual cortices and vestibular cortex for self-motion perception (Brandt et al., 1998; Kleinschmidt et al., 2002; Smith et al., 2012; Frank et al., 2014, 2016). In addition, we observed significant changes in functional connectivity in the higher-order visual areas of the inferior and middle temporal gyri during recovery from VIMS (Supplementary Figure $1 \mathrm{~g}$ and $1 \mathrm{~m}$; Tables 3,4 , and 5). However, no change in connectivity was seen in the PIVC, known as the centre of the neural basis of vestibular sensation. This might have been because visual inputs could be the only trigger of VIMS and because the visual system could be the sole centre of brain dynamics during the recovery phase.

Finally, the third possibility is that the increased functional connectivity in these brain regions during recovery from VIMS might reflect the maintenance of spatial memory. We 
1 found increased connectivity between the cerebellar tonsil and parahippocampal gyrus

2 (Supplementary Figure 1h). The parahippocampal gyrus has been suggested to be the

3 centre of visual spatial information processing (Epstein et al., 1998; Epstein, 2008) and

4 is presumably related to the recognition and memorisation of the space where one exists.

5 Previous resting-state fMRI studies of memory maintenance reported a significant

6 increase in functional connectivity between the brain regions involved in memory

7 processing after memory tasks (Tambini et al., 2010; Gordon et al., 2014). For example,

8 in Tambini et al. (2010), the resting-state connectivity between the hippocampus and

9 lateral occipital complex increased after associative memory tasks, which enabled the

10 prediction of subsequent memory performance. Therefore, the increased connectivity in

11 these regions might reflect novel spatial experiences in memory processing in the present

12 participants with VIMS.

13 If change in functional connectivity is related to the neuroplastic changes and/or 14 memory processing described above, then our findings might provide some insight into 15 the neural process underlying the sensory conflict hypothesis (Reason \& Brand, 1975; 16 Reason, 1978; Oman, 1990). This hypothesis suggests that a conflict or mismatch among 17 multiple sensory inputs, including visual and vestibular inputs, would cause MS. 18 Interestingly, this hypothesis also indicates that a conflict not only among sensory inputs, 19 but also between a sensory input and an empirically acquired sensory memory (so-called 20 'neural store') would cause MS. From this perspective, individuals with MS such as 21 VIMS can develop adaptation, and tolerance increases when similar stimuli are repeated 22 because of the reduced conflict between a sensory input and the 'neural store', which is 23 the memory or plasticity acquired from the repeated sensory experience (Reason \& Brand, 1975; Reason, 1978; Oman, 1990). Although the 'neural store' has been just a concept, 25 the actual brain network associated with this concept may have been revealed by our 26 findings. If so, these findings can advance our understanding of the neural mechanisms underlying the development of MS and adaptation to it.

\section{Competing Interests}

30 The authors declare that they have no conflict of interest related to this publication.

\section{Author Contributions}


1 J.M.: Conceptualisation, Formal analysis, Investigation, Writing - Original Draft, Writing 2 - Review \& Editing.

3 H.Y.: Project Administration, Supervision, Conceptualisation, Methodology, Writing 4 Original Draft, Writing - Review \& Editing, Funding acquisition.

5 Y.I.: Supervision, Writing - Review.

6 H.Y., T.Y.: Methodology, Software support, Writing - Review.

7 T.M., M.U., T.H.: Methodology, Imaging, Writing - Review.

8

\section{Data Accessibility}

10 Data in this study are available upon request. Please contact the corresponding author for 11 access.

12

\section{Abbreviations}

14 VIMS, visually induced motion sickness; MS, motion sickness; MRI, magnetic resonance 15 imaging; fMRI, functional magnetic resonance imaging; SSQ, Simulator Sickness 16 Questionnaire, ROI, region of interest; MT+, middle temporal complex. 


\section{References}

Albert NB, Robertson EM, Mehta P, Miall RC (2009a) Resting state networks and memory consolidation. Communicative \& integrative biology 2:530-532

Albert NB, Robertson EM, Miall RC (2009b) The Resting Human Brain and Motor Learning. Current Biology 19:1023-1027 doi: https://doi.org/10.1016/j.cub.2009.04.028

Bates D, Maechler M, Bolker B, Walker S (2015) lme4: Linear mixed-effects models using Eigen and S4. Journal of Statistical Software 67:1-48

Brandt T, Bartenstein P, Janek A, Dieterich M (1998) Reciprocal inhibitory visualvestibular interaction. Visual motion stimulation deactivates the parieto-insular vestibular cortex. Brain 121:1749-1758 doi: 10.1093/brain/121.9.1749 \%J Brain

Corbetta M, Akbudak E, Conturo TE, et al. (1998) A common network of functional areas for attention and eye movements. Neuron 21:761-773

Cox RW (1996) AFNI: software for analysis and visualization of functional magnetic resonance neuroimages. Computers and Biomedical research 29:162-173

Craig AD (2002) How do you feel? Interoception: the sense of the physiological condition of the body. Nature reviews neuroscience 3:655

Craig AD, Craig AD (2009) How do you feel--now? The anterior insula and human awareness. Nature reviews neuroscience 10

Crick FC, Koch C (2005) What is the function of the claustrum? Philosophical Transactions of the Royal Society of London B: Biological Sciences 360:12711279

Dieterich M, Bense S, Stephan T, Yousry TA, Brandt T (2003) fMRI signal increases and decreases in cortical areas during small-field optokinetic stimulation and central fixation. Experimental Brain Research 148:117-127

Dieterich M, Bucher SF, Seelos KC, Brandt T (1998) Horizontal or vertical optokinetic stimulation activates visual motion-sensitive, ocular motor and vestibular cortex areas with right hemispheric dominance. An fMRI study. Brain: a journal of neurology 121:1479-1495

Ebenholtz SM (1992) Motion sickness and oculomotor systems in virtual environments. Presence: Teleoperators \& Virtual Environments 1:302-305

Ebenholtz SM, Cohen MM, Linder BJ (1994) The possible role of nystagmus in motion sickness: a hypothesis. Aviation, space, and environmental medicine 65:10321035

Epstein R, Kanwisher N (1998) A cortical representation of the local visual environment. Nature 392:598

Epstein RA (2008) Parahippocampal and retrosplenial contributions to human spatial navigation. Trends in cognitive sciences 12:388-396

Farmer AD, Ban VF, Coen SJ, et al. (2015) Visually induced nausea causes characteristic changes in cerebral, autonomic and endocrine function in humans. The Journal of physiology 593:1183-1196

Frank SM, Baumann O, Mattingley JB, Greenlee MW (2014) Vestibular and visual responses in human posterior insular cortex. Journal of neurophysiology 112:2481-2491

Frank SM, Wirth AM, Greenlee MW (2016) Visual-vestibular processing in the human Sylvian fissure. Journal of neurophysiology 116:263-271

Friston KJ, Frith CD, Liddle PF, Frackowiak RSJ (1993) Functional connectivity: the principal-component analysis of large (PET) data sets. Journal of Cerebral Blood 
Flow \& Metabolism 13:5-14

Gordon EM, Breeden AL, Bean SE, Vaidya CJ (2014) Working memory- related changes in functional connectivity persist beyond task disengagement. Human brain mapping 35:1004-1017

Gotts SJ, Simmons WK, Milbury LA, Wallace GL, Cox RW, Martin A (2012) Fractionation of social brain circuits in autism spectrum disorders. Brain 135:2711-2725

Hahsler M, Hornik K, Buchta C (2008) Getting things in order: an introduction to the R package seriation. Journal of Statistical Software 25:1-34

Kennedy RS, Drexler J, Kennedy RC (2010) Research in visually induced motion sickness. Applied ergonomics 41:494-503

Kennedy RS, Fowlkes JE, Lilienthal MG (1993a) Postural and performance changes following exposures to flight simulators. Aviation, Space, and Environmental Medicine

Kennedy RS, Lane NE, Berbaum KS, Lilienthal MG (1993b) Simulator sickness questionnaire: An enhanced method for quantifying simulator sickness. The international journal of aviation psychology 3:203-220

Kleinschmidt A, Thilo KV, Büchel C, Gresty MA, Bronstein AM, Frackowiak RSJ (2002) Neural correlates of visual-motion perception as object-or self-motion. Neuroimage 16:873-882

Klosterhalfen S, Pan F, Kellermann S, Enck P (2006) Gender and race as determinants of nausea induced by circular vection. Gender medicine 3:236-242

Konen CS, Kleiser R, Seitz RJ, Bremmer F (2005) An fMRI study of optokinetic nystagmus and smooth-pursuit eye movements in humans. Experimental Brain Research 165:203-216

Koslucher F, Haaland E, Malsch A, Webeler J, Stoffregen TA (2015) Sex differences in the incidence of motion sickness induced by linear visual oscillation. Aerospace medicine and human performance 86:787-793

Kuznetsova A, Brockhof BP, Christensen HBR (2017) lmerTest: Tests in Linear Mixed Effects Models. Journal of Statistical Software 82:1-26

Lewis CM, Baldassarre A, Committeri G, Romani GL, Corbetta M (2009) Learning sculpts the spontaneous activity of the resting human brain. Proceedings of the National Academy of Sciences:pnas-0902455106

Miyazaki J, Yamamoto H, Ichimura Y, et al. (2015) Inter-hemispheric desynchronization of the human MT+ during visually induced motion sickness. Experimental brain research 233:2421-2431

Munafo J, Diedrick M, Stoffregen TA (2017) The virtual reality head-mounted display Oculus Rift induces motion sickness and is sexist in its effects. Experimental brain research 235:889-901

Napadow V, Sheehan JD, Kim J, et al. (2012) The brain circuitry underlying the temporal evolution of nausea in humans. Cerebral cortex 23:806-813

Oman CM (1990) Motion sickness: a synthesis and evaluation of the sensory conflict theory. Canadian journal of physiology and pharmacology 68:294-303

Peltier SJ, LaConte SM, Niyazov DM, Liu JZ, Sahgal V, Yue GH, Hu XP (2005) Reductions in interhemispheric motor cortex functional connectivity after muscle fatigue. Brain research 1057:10-16

Reason JT (1978) Motion sickness adaptation: a neural mismatch model. Journal of the Royal Society of Medicine 71:819-829 
Reason JT, Brand JJ (1975) Motion sickness. Academic press

Riccio GE, Stoffregen TA (1991) An ecological theory of motion sickness and postural instability. Ecological psychology 3:195-240

Shupak A, Gordon CR (2006) Motion sickness: advances in pathogenesis, prediction, prevention, and treatment. Aviation, space, and environmental medicine 77:12131223

Smith AT, Wall MB, Thilo KV (2011) Vestibular inputs to human motion-sensitive visual cortex. Cerebral cortex 22:1068-1077

Stanney K, Salvendy G (1998) Aftereffects and sense of presence in virtual environments: Formulation of a research and development agenda. International Journal of Human-Computer Interaction 10:135-187

Stevens WD, Buckner RL, Schacter DL (2009) Correlated low-frequency BOLD fluctuations in the resting human brain are modulated by recent experience in category-preferential visual regions. Cerebral cortex 20:1997-2006

Stevens WD, Tessler MH, Peng CS, Martin A (2015) Functional connectivity constrains the category- related organization of human ventral occipitotemporal cortex. Human brain mapping 36:2187-2206

Stoffregen TA, Chang C-H, Chen F-C, Zeng W-J (2017) Effects of decades of physical driving on body movement and motion sickness during virtual driving. PLoS one 12:e0187120

Suzuki R, Shimodaira H (2019) pvclust: Hierarchical Clustering with P-Values via Multiscale Bootstrap Resampling. Bioinformatics 22:1540-1542 doi: 10.1093/bioinformatics/btl117

Tambini A, Ketz N, Davachi L (2010) Enhanced brain correlations during rest are related to memory for recent experiences. Neuron 65:280-290

Team RC (2016) R: A language and environment for statistical computing. Vienna: R Foundation for Statistical Computing; 2014. In:

Toschi N, Kim J, Sclocco R, Duggento A, Barbieri R, Kuo B, Napadow V (2017) Motion sickness increases functional connectivity between visual motion and nauseaassociated brain regions. Autonomic Neuroscience 202:108-113

Ujike H, Ukai K, Nihei K (2008) Survey on motion sickness-like symptoms provoked by viewing a video movie during junior high school class. Displays 29:81-89

Van Marle HJF, Hermans EJ, Qin S, Fernández G (2010) Enhanced resting-state connectivity of amygdala in the immediate aftermath of acute psychological stress. Neuroimage 53:348-354

Vohn R, Fimm B, Weber J, et al. (2007) Management of attentional resources in withinmodal and cross- modal divided attention tasks: An fMRI study. Human brain mapping 28:1267-1275

Wall MB, Smith AT (2008) The representation of egomotion in the human brain. Current biology 18:191-194

Walter HJ, Li R, Munafo J, Curry C, Peterson N, Stoffregen TA (2019) Unstable coupling of body sway with imposed motion precedes visually induced motion sickness. Human Movement Science 64:389-397 
1 Table Captions

2

3

4

5 6

7 This analysis was performed using a linear mixed-effects model with a within-participants

8 fixed effect PHASE (Rest-1 vs. Rest-2 vs. Rest-3), a between-participants fixed effect

9 GROUP (VIMS vs. the healthy participant group), an interaction of PHASE $\times$ GROUP, 10

Table 1. Statistical analysis of the degree of recovery in the subjective score of the Simulator Sickness Questionnaire (SSQ) (decrease in the SSQ between the post- and pre-rest phases) and a random effect of each individual participant. If there was a PHASE $\times$ GROUP interaction, post hoc analysis was conducted on the simple effect of PHASE. For the effect size of the interaction and the simple effect, $f$-squared and Cohen's $d$ were computed, respectively. If there was no interaction, the main effect of PHASE was tested. The $P$ values of the interaction were Bonferroni corrected for the number of scales of the SSQ, whereas those of the post hoc analyses were corrected for the number of the SSQ scales, participant groups, and comparison repetitions.

Table 2. Brain regions whose connectedness increased significantly during the recovery phase from visually induced motion sickness

Connectedness was computed using the AFNI software 3dTCorrMap function separately for the 12 participants. These data were then transformed to the standard Talairach space. In this standard space, the connectedness was compared by use of the AFNI $3 d L M E$ function, and the brain regions whose connectedness changed significantly during the recovery from VIMS were detected. Voxel-wise threshold $P<0.0001$ and cluster level threshold $\alpha<0.10$ were used.

Table 3. Brain region pairs whose functional connectivity increased during the recovery phase of visually induced motion sickness (VIMS) and the GROUP $\times$ PHASE interaction effect

A seed-based functional connectivity analysis revealed 19 brain region pairs whose 
1 connectivities increased during the recovery from VIMS. The increases in connectivity were statistically tested with a linear mixed-effects model. The linear mixed-effects model had a within-participants fixed effect PHASE (Rest-1 vs. Rest-2 vs. Rest-3), a betweenparticipants fixed effect GROUP (VIMS $v s$. the healthy participant group), interaction of PHASE $\times$ GROUP, and a random effect of each individual participant. For the effect size of the PHASE $\times$ GROUP interaction, $f$-squared was computed. The $P$ values of the interaction were Bonferroni corrected for the number of brain region pairs. The numbering of the 19 pairs corresponds to that in Figure 4 and Supplementary Figure 1.

Table 4. Statistical analysis of the functional connectivity of brain region pairs for the visually induced motion sickness (VIMS) group

The linear mixed-effects model had a within-participants fixed effect PHASE (Rest-1 vs.

Table 5. Statistical analysis of the functional connectivity of brain region pairs for the healthy group

The description is the same as that of Table 4 but for the healthy participant group.

Supplementary Table 1. Statistical analysis of the effect of head movement on functional connectivity

The effect of head motion (maximum framewise displacement [FD]) on functional connectivity was statistically assessed by using a linear mixed-effects model, in which a within-participants fixed effect of FD and interactions of FD $\times$ PHASE and FD $\times$ 
1 GROUP were included, in addition to the fixed effects of PHASE, GROUP, and PHASE

$2 \times$ GROUP and a random effect of each individual participant. If the head movement-

3 related interactions (i.e., FD $\times$ PHASE and FD $\times$ GROUP) were statistically significant, 4 the statistics for FD were omitted because it was difficult to determine the effect. 
1 Figure Captions

2

3 Figure 1. Schematic diagram showing the time course of the experimental session 4

5 After the first rest phase (Rest-1), the local motion stimulus was presented as a control. 6 After the second rest phase (Rest-2), the global motion stimulus, which can induce 7 visually induced motion sickness (VIMS), was presented. Finally, the third rest phase 8 (Rest-3) comprised the recovery from VIMS.

Figure 2. Time course of the change in the Total Score (TS) of the Simulator Sickness Questionnaire (SSQ)

The vertical axis shows the change in the TS (post-score minus pre-score) of the SSQ.

4 The horizontal axis shows the experimental phase (Rest-1, Rest-2, and Rest-3). The visually induced motion sickness (VIMS) group's participants experienced motion sickness soon before the third rest phase (Rest-3), and recovery from symptoms occurred during Rest-3. Accordingly, the score of the VIMS group (solid line) significantly decreased before and after Rest-3, whereas the score of the healthy group participants (broken line), who did not experience VIMS, did not change as much.

Figure 3. Twelve brain regions whose connectedness significantly increased during the recovery phase from visually induced motion sickness (VIMS)

Each row is as follows: a, left thalamus; b, anterior cingulate; c, left insula; d, right insula; e, cerebellar tonsil; f, claustrum; g, right lingual gyrus; h, cingulate gyrus; i, right thalamus; j, left superior frontal gyrus; k, left lingual gyrus; and 1, inferior frontal gyrus. Each column shows, from left to right, axial, sagittal, and coronal brain images. The following thresholds were set: voxel-wise threshold $P<0.0001$, cluster level threshold $\alpha$ $<0.10$.

Figure 4. Brain regions whose functional connectivity with the seed regions increased in the recovery phase from visually induced motion sickness (VIMS) 
1

2 Each column shows, from left to right, axial, sagittal, and coronal brain images, and the

3 change in the functional connectivity ( $\mathrm{Z}$ scores averaged within the participants' groups)

4 of each experimental phase, separately for VIMS and healthy groups, respectively (error

5 bars are standard errors). Each panel is the result for the following brain regions (the brain

6 region in brackets is the corresponding seed): a-left superior temporal gyrus (right insula),

7 b-right inferior parietal lobule (right claustrum), c-left superior temporal gyrus (right

8 claustrum), d-left inferior parietal lobule (right claustrum); and e-left lentiform nucleus

9 (left superior frontal gyrus). For this mapping, we used the following thresholds: voxel-

10 wise threshold $P<0.0001$, cluster level threshold $\alpha<0.05$. The numbering of brain region

11 pairs corresponds to that in Tables 3, 4, and 5.

12

13 Figure 5. Correlation plots between the change in the Total Score (TS) of the 14 Simulator Sickness Questionnaire (SSQ) and the change in functional connectivity 15

The vertical axis is the change in the TS of the SSQ: Rest-3 minus the average of Rest-1 and Rest-2; the greater the recovery in VIMS symptoms, the greater the decrease in this score. The horizontal axis is the change in functional connectivity: Rest-3 minus the average of Rest-1 and Rest-2. In the graphs, $\rho$ indicates Spearman's rank correlation coefficient, with the corresponding $P$ value shown below. The $P$ values were Bonferroni corrected for multiple comparisons among the 19 brain region pairs. Abbreviations: IPL, inferior parietal lobule; L, left; R, right; SFG, superior frontal gyrus; STG, superior temporal gyrus.

Figure 6. Hierarchical clustering trees of the 12 brain regions showing recoveryselective increases in connectedness

(a) The dendrogram of the VIMS group for the recovery phase (Rest-3), which was derived from the distance matrix (Supplementary Fig. 2a), whose elements indicate 1 minus absolute partial correlation between each pair of the 12 regions. Statistically significant clusters are marked with an asterisk $(P<0.05)$ or plus $(P<0.1)$. Brain regions showing recovery-selective increases in the ROI-based functional connectivity and 
1 correlations with SSQ are underlined and in italics, respectively. (b) The same as (a) but

2 for the healthy group. (c) The same as (a) but for the control phase (the average of Rest-

31 and Rest-2). (d) The same as (c) but for the healthy group. Abbreviations: L, left; R,

4 right; LG, lingual gyrus; SFG, superior frontal gyrus; IFG, inferior frontal gyrus.

6 Supplementary Figure 1. Brain regions whose functional connectivity with the seed 7 regions increased in the recovery phase from visually induced motion sickness 8 (VIMS)

10 Each column shows, from left to right, axial, sagittal, and coronal brain images, and the 11 change in functional connectivity ( $Z$ scores averaged within the participants' groups) of 12 each experimental phase, separately for VIMS and healthy groups, respectively (error 13 bars are standard errors). Each panel is the result for the following brain regions (the brain 14 region in brackets is the corresponding seed): a-right insula (right thalamus), b-right 15 inferior parietal lobule (right thalamus), c-right declive (right thalamus), d-left thalamus (left insula), e-right inferior parietal lobule (left insula), f-left thalamus (right insula), g17 left inferior temporal gyrus (right insula), h-left parahippocampal gyrus (left cerebellar tonsil), i-left cingulate gyrus (right claustrum), j-left inferior frontal gyrus (right

19 claustrum), k-right insula (left cingulate gyrus), l-left medial frontal gyrus (left superior 20 frontal gyrus), m-left middle temporal gyrus (left superior frontal gyrus), and n-left 21 inferior frontal gyrus (right inferior frontal gyrus). For this mapping, we used the 22 following thresholds: voxel-wise threshold $P<0.0001$, cluster level threshold $\alpha<0.05$. 23 The numbering of brain region pairs corresponds to that in Tables 3, 4, and 5.

Supplementary Figure 2. Distance matrices of the 12 brain regions showing 26 recovery-selective increases in connectedness

(a) The distance matrix of the VIMS group for the recovery phase (Rest-3), whose 30 Brain regions showing recovery-selective increases in the ROI-based functional connectivity and correlations with SSQ are underlined and in italics, respectively. (b) The same as (a) but for the healthy group. (c) The same as (a) but for the control phase (the 
1 average of Rest-1 and Rest-2). (d) The same as (c) but for the healthy group.

2 Abbreviations: L, left; R, right; LG, lingual gyrus; SFG, superior frontal gyrus; IFG,

3 inferior frontal gyrus.

4

5 Supplementary Figure 3. Statistically independent brain networks related to 6 recovery from visually induced motion sickness (VIMS)

8 (a) The within-participant averaged distance matrix for the recovery phase of the VIMS

9 group. Each element represents a cross-correlation coefficient between each pair of the 1022 regions determined by a dictionary learning technique. This analysis derived 22 11 regions of interest (ROIs). (b) The top 10\% connections derived from the distance matrix. 12 The connections are overlaid on a glass brain. Yellow circles and red lines denote the 13 derived regions and connections, respectively. The connections are as follows: R Posterior 14 Cingulate-L Lingual Gyrus, L Lingual Gyrus-R MOG, L Lingual Gyrus-L MTG, L 15 Lingual Gyrus-R MOG, L Lingual Gyrus-L MOG, R Superior Occipital Gyrus-R MOG, 16 R Culmen-R Parahippocampal Gyrus, R Culmen-R Cuneus, R Culmen-L Cuneus, R 17 Culmen-R MOG, R Culmen-L MOG, R Parahippocampal Gyrus-L Cuneus, R 18 Parahippocampal Gyrus-L Posterior Cingulate, R Cuneus-L Cuneus, R Cuneus-L 19 Precuneus, L Cuneus-R MOG, R MOG-L MTG, L MOG-R MOG, L MOG-R MOG, L 20 MTG-R MOG, L MTG-L MOG, and R MOG-R MOG. Abbreviations: L, left; R, right; 21 MTG, middle temporal gyrus; MOG, middle occipital gyrus. 
Table 1. Statistical analysis of the degree of recovery in the subjective score of the Simulator Sickness Questionnaire (SSQ) (decrease in the SSQ between the post- and pre-rest phases)

\begin{tabular}{|c|c|c|c|c|c|c|c|c|c|c|}
\hline \multirow{3}{*}{ Scale } & \multicolumn{2}{|c|}{ GROUP $\times$ PHASE interaction } & & \multicolumn{7}{|c|}{ Simple effect of PHASE } \\
\hline & \multirow{2}{*}{$\begin{array}{c}\text { Statistics } \\
P \text { value }\left(\chi^{2}\right)\end{array}$} & \multirow{2}{*}{$\begin{array}{c}\text { Effect size } \\
\qquad f^{2}\end{array}$} & & \multicolumn{3}{|c|}{ VIMS } & & \multicolumn{3}{|c|}{ Healthy } \\
\hline & & & & $\begin{array}{c}\text { Coefficient } \\
\text { Estimate } \pm \text { SE }\end{array}$ & $\begin{array}{c}\text { Statistics } \\
P \text { value }\left(\chi^{2}\right)\end{array}$ & $\begin{array}{l}\text { Effect size } \\
\text { Cohen's } d\end{array}$ & & $\begin{array}{c}\text { Coefficient } \\
\text { Estimate } \pm \mathrm{SE}\end{array}$ & $\begin{array}{c}\text { Statistics } \\
P \text { value }\left(\chi^{2}\right)\end{array}$ & $\begin{array}{l}\text { Effect size } \\
\text { Cohen's } d\end{array}$ \\
\hline \multirow{3}{*}{ Total score } & & & Rest-1 vs. Rest-2 & $46.75 \pm 11.45$ & $>1(0.03)$ & 0.07 & & $-4.99 \pm 2.29$ & $.417(4.72)$ & 0.89 \\
\hline & $.005(13.30)$ & 0.45 & Rest- 2 vs. Rest- 3 & $-41.76 \pm 10.77$ & $.001(15.04)$ & 1.58 & & $-0.62 \pm 2.29$ & $>1(0.07)$ & 0.11 \\
\hline & & & Rest-1 vs. Rest-3 & $-43.63 \pm 10.77$ & $0.0007(16.41)$ & 1.65 & & $-5.61 \pm 2.29$ & $.203(5.98)$ & 1.00 \\
\hline \multirow{3}{*}{ Disorientation } & & & Rest-1 vs. Rest-2 & $-2.32 \pm 15.09$ & $>1(0.02)$ & 0.06 & & $-2.32 \pm 3.19$ & $>1(0.53)$ & 0.30 \\
\hline & $.0002(19.16)$ & 0.72 & Rest- 2 vs. Rest-3 & $-64.96 \pm 15.09$ & $0.0002(18.52)$ & 1.76 & & $2.32 \pm 3.19$ & $>1(<0.01)$ & 0.30 \\
\hline & & & Rest-1 vs. Rest-3 & $-67.28 \pm 15.09$ & $0.0001(19.87)$ & 1.82 & & $-5.92 \pm 3.19$ & $>1(0.52)$ & $<0.01$ \\
\hline & & & \multicolumn{8}{|c|}{ Main effect of PHASE } \\
\hline & & & \multicolumn{3}{|c|}{$\begin{array}{c}\text { Coefficient } \\
\text { Estimate } \pm \text { SE }\end{array}$} & & $\begin{array}{c}\text { Statistics } \\
P \text { value }(\chi 2)\end{array}$ & \multicolumn{3}{|c|}{$\begin{array}{l}\text { Effect size } \\
\text { Cohen's d }\end{array}$} \\
\hline Nausea & $.084(7.74)$ & 0.24 & \multicolumn{3}{|c|}{$-7.16 \pm 3.46$} & & $.539(4.28)$ & \multicolumn{3}{|c|}{0.6} \\
\hline Oculomotor & $.259(5.48)$ & 0.16 & \multicolumn{3}{|c|}{$-4.21 \pm 1.96$} & & $.444(4.62)$ & \multicolumn{3}{|c|}{0.62} \\
\hline
\end{tabular}

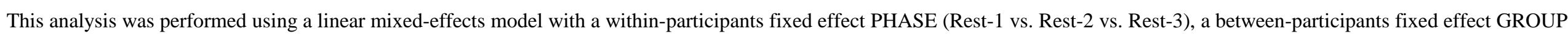

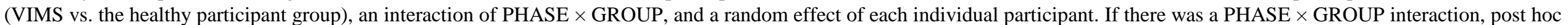

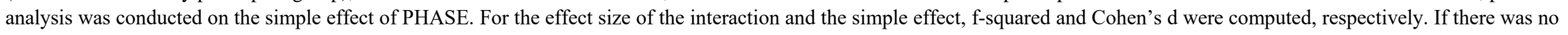

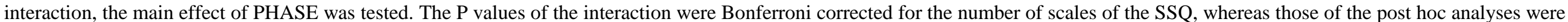


Table 2. Brain regions whose connectedness increased significantly during the recovery phase from visually induced motion sickness

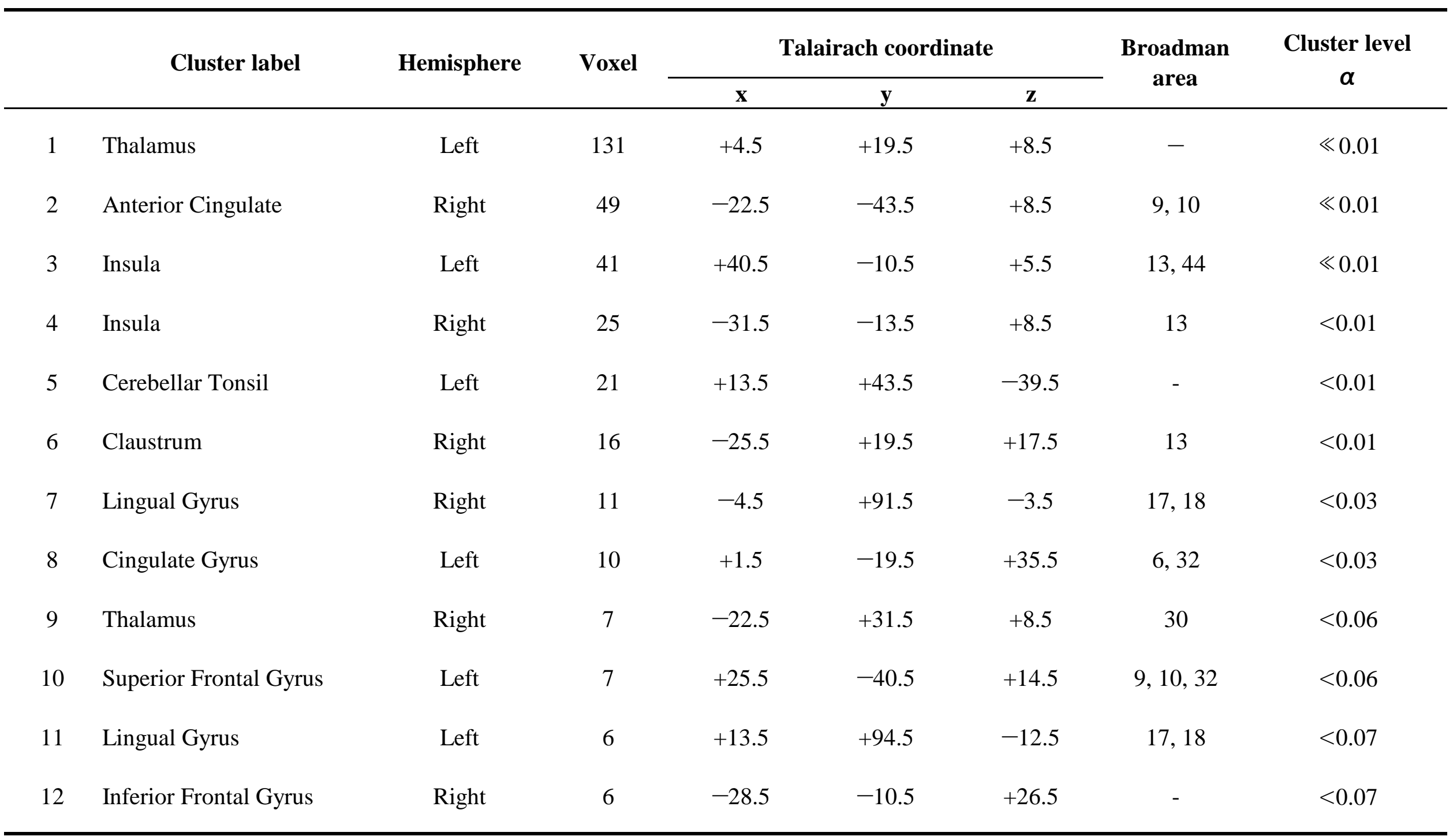

Connectedness was computed using the AFNI software 3dTCorrMap function separately for the 12 participants. These data were then transformed to the standard Talairach space. In this standard space, the connectedness was compared by use of the AFNI 3dLME function, and the brain regions whose connectedness changed significantly during the recovery from VIMS were detected. Voxel-wise threshold P $<0.0001$, cluster level threshold $\alpha<0.10$ were used. 


\begin{tabular}{|c|c|c|c|c|c|c|c|c|c|}
\hline \multirow{3}{*}{ Seed label } & \multirow{3}{*}{ Cluster label } & \multirow{3}{*}{ Hemisphere } & \multicolumn{2}{|c|}{ GROUP $\times$ PHASE interaction } & \multirow{3}{*}{ Voxel } & \multirow{2}{*}{\multicolumn{3}{|c|}{ Talairach coordinate }} & \multirow{3}{*}{ Broadman area } \\
\hline & & & \multirow{2}{*}{$\begin{array}{l}\text { Test statistics } \\
P \text { value }\left(\chi^{2}\right)\end{array}$} & \multirow{2}{*}{$\begin{array}{l}\text { Effect size } \\
\qquad f^{2}\end{array}$} & & & & & \\
\hline & & & & & & $\mathbf{x}$ & $\mathbf{y}$ & $\mathbf{z}$ & \\
\hline \multirow{3}{*}{ L Thalamus } & 1 Insula & Right & $.003(17.45)$ & 0.11 & 21 & -34.5 & -13.5 & +2.5 & 13,47 \\
\hline & 2 Inferior Parietal Lobule & Right & $.014(14.48)$ & 0.12 & 9 & -55.5 & +28.5 & +26.5 & $2,13,40$ \\
\hline & 3 Declive & Right & $.0003(20.12)$ & 0.10 & 8 & -4.5 & +58.5 & -12.5 & - \\
\hline \multirow{2}{*}{ L Insula } & 4 Thalamus & Left & $.001(18.99)$ & 0.11 & 15 & +4.5 & +10.5 & +11.5 & - \\
\hline & 5 Inferior Parietal Lobule & Right & $.004(16.93)$ & 0.28 & 9 & -58.5 & +28.5 & +29.5 & 2,40 \\
\hline \multirow{3}{*}{$\mathrm{R}$ Insula } & 6 Thalamus & Left & $5 \times 10^{-5}(25.78)$ & 0.15 & 23 & +7.5 & +16.5 & +5.5 & - \\
\hline & 7 Superior Temporal Gyrus & Left & $.002(18.81)$ & 0.16 & 13 & +55.5 & +31.5 & +11.5 & $22,41,42$ \\
\hline & 8 Inferior Temporal Gyrus & Left & $.001(19.26)$ & 0.21 & 9 & +49.5 & +58.5 & -9.5 & $19,20,37$ \\
\hline L Cerebellar Tonsil & 9 Parahippocampal Gyrus & Left & $.005(16.37)$ & 0.18 & 11 & +16.5 & +16.5 & -15.5 & - \\
\hline \multirow{5}{*}{ R Claustrum } & 10 Cingulate Gyrus & Left & $.0004(21.52)$ & 0.27 & 83 & +1.5 & -22.5 & +26.5 & 24,32 \\
\hline & 11 Inferior Parietal Lobule & Right & $.002(18.72)$ & 0.23 & 22 & -55.5 & +31.5 & +26.5 & $13,40,42$ \\
\hline & 12 Inferior Frontal Gyrus & Left & $.003(17.45)$ & 0.24 & 17 & +40.5 & -25.5 & +2.5 & $13,45,47$ \\
\hline & 13 Superior Temporal Gyrus & Left & $.0002(23.07)$ & 0.21 & 11 & +58.5 & +25.5 & +11.5 & $22,40,41,42$ \\
\hline & 14 Inferior Parietal Lobule & Left & .004 (16.95) & 0.13 & 8 & +55.5 & +34.5 & +23.5 & $13,40,42$ \\
\hline \multirow[t]{2}{*}{ L Cingulate Gyrus } & 15 Insula & Right & $5 \times 10^{-5}(25.62)$ & 0.23 & 19 & -25.5 & +25.5 & +20.5 & 13 \\
\hline & 16 Medial Frontal Gyrus & Left & $5 \times 10^{-5}(25.53)$ & 0.20 & 67 & +4.5 & -25.5 & +41.5 & 8,32 \\
\hline \multirow[t]{2}{*}{ L Superior Frontal Gyrus } & 17 Middle Temporal Gyrus & Left & $.004(17.16)$ & 0.30 & 12 & +46.5 & +52.5 & -0.5 & 19,37 \\
\hline & 18 Lentiform Nucleus & Left & $.0001(17.72)$ & 0.29 & 8 & +28.5 & +19.5 & +11.5 & 13 \\
\hline R Inferior Frontal Gyrus & 19 Inferior Frontal Gyrus & Left & $.002(18.80)$ & 0.31 & 14 & +40.5 & -28.5 & -0.5 & $13,45,47$ \\
\hline
\end{tabular}

A seed-based functional connectivity analysis revealed 19 brain region pairs whose connectivities increased during the recovery from VIMS. The increases in connectivity were statistically tested with a linear mixed-effects A seed-based functional
model. The linear mixed-effects model had a within-participants fixed effect PHASE (Rest-1 vs. Rest-2 vs. Rest-3), a between-participants fixed effect GROUP (VIMS vs. the healthy participant group), interaction of PHASE $\times$ GROUP, and a random effect of each individual participant. For the effect size of the PHASE $\times$ GROUP interaction, f-squared was computed. The P values of the interaction were Bonferroni corrected for the PHASE $\times$ GROUP, and a random effect of each individual participant. For the effect size of the PHASE $\times$ GROUP inter
number of brain region pairs. The numbering of the 19 pairs corresponds to that in Figure 4 and Supplementary Figure 1 . 


\begin{tabular}{|c|c|c|c|c|c|c|c|c|c|c|c|}
\hline & & & \multicolumn{3}{|c|}{ Rest-1 vs. Rest-2 } & \multicolumn{3}{|c|}{ Rest-2 vs. Rest-3 } & \multicolumn{3}{|c|}{ Rest-1 vs. Rest-3 } \\
\hline & & & $\begin{array}{c}\text { Coefficient } \\
\text { Estimate } \pm \text { SE }\end{array}$ & $\begin{array}{l}\text { Test statistics } \\
P \text { value }\left(\gamma^{2}\right)\end{array}$ & $\begin{array}{l}\text { Effect size } \\
\text { Cohen's } d \\
\end{array}$ & $\begin{array}{c}\text { Coefficient } \\
\text { Estimate } \pm \text { SE }\end{array}$ & $\begin{array}{c}\text { Test statistics } \\
P \text { value }\left(\gamma^{2}\right) \\
\end{array}$ & $\begin{array}{l}\text { Effect size } \\
\text { Cohen's } d\end{array}$ & $\begin{array}{c}\text { Coefficient } \\
\text { Estimate } \pm \text { SE }\end{array}$ & $\begin{array}{l}\text { Test statistics } \\
P \text { value }\left(\gamma^{2}\right) \\
\end{array}$ & $\begin{array}{l}\text { Effect size } \\
\text { Cohen's } d \\
\end{array}$ \\
\hline \multirow{3}{*}{ L Thalamus } & 1 Insula & Right & $-0.07 \pm 0.07$ & $>1(1.05)$ & 0.42 & $0.34 \pm 0.07$ & $5 \times 10^{-5}(25.70)$ & 2.07 & $0.27 \pm 0.07$ & $.006(16.37)$ & 1.65 \\
\hline & 2 Inferior Parietal Lobule & Right & $-0.04 \pm 0.06$ & $>1(0.44)$ & 0.27 & $0.30 \pm 0.06$ & $4 \times 10^{-5}(25.94)$ & 2.08 & $0.26 \pm 0.06$ & $.001(19.61)$ & 1.81 \\
\hline & 3 Declive & Right & $-0.03 \pm 0.04$ & $>1(0.82)$ & 0.37 & $0.27 \pm 0.04$ & $3 \times 10^{-12}(57.83)$ & 3.10 & $0.24 \pm 0.04$ & $2 \times 10^{-9}(44.86)$ & 2.73 \\
\hline \multirow{2}{*}{ L Insula } & 4 Thalamus & Left & $0.02 \pm 0.05$ & $>1(0.10)$ & 0.13 & $0.27 \pm 0.05$ & $2 \times 10^{-6}(32.17)$ & 2.32 & $0.29 \pm 0.05$ & $2 \times 10^{-7}(35.93)$ & 2.45 \\
\hline & 5 Inferior Parietal Lobule & Right & $-0.04 \pm 0.07$ & $>1(0.35)$ & 0.24 & $0.43 \pm 0.07$ & $8 \times 10^{-8}(38.05)$ & 2.52 & $0.39 \pm 0.07$ & $3 \times 10^{-6}(31.12)$ & 2.28 \\
\hline \multirow{3}{*}{ R Insula } & 6 Thalamus & Left & $-0.06 \pm 0.05$ & $>1(1.39)$ & 0.48 & $0.40 \pm 0.05$ & $2 \times 10^{-11}(53.99)$ & 3.00 & $0.34 \pm 0.05$ & $8 \times 10^{-8}(38.05)$ & 2.52 \\
\hline & 7 Superior Temporal Gyrus & Left & $0.11 \pm 0.08$ & $>1(2.13)$ & 0.60 & $0.30 \pm 0.08$ & $.010(15.40)$ & 1.60 & $0.41 \pm 0.08$ & $.0003(29.00)$ & 2.20 \\
\hline & 8 Inferior Temporal Gyrus & Left & $0.11 \pm 0.07$ & $>1(2.79)$ & 0.68 & $0.26 \pm 0.07$ & $.016(14.53)$ & 1.56 & $0.38 \pm 0.07$ & $5 \times 10^{-6}(30.05)$ & 2.24 \\
\hline \multirow[t]{3}{*}{ L Cerebellar Tonsil } & 9 Parahippocampal Gyrus & Left & $-0.003 \pm 0.08$ & $>1(0.001)$ & 0.01 & $0.32 \pm 0.08$ & $.008(15.92)$ & 1.63 & $0.32 \pm 0.08$ & $.009(15.65)$ & 1.62 \\
\hline & 10 Cingulate Gyrus & Left & $-0.01 \pm 0.06$ & $>1(0.05)$ & 0.09 & $0.33 \pm 0.06$ & $3 \times 10^{-6}(31.22)$ & 2.28 & $0.32 \pm 0.06$ & $9 \times 10^{-6}(28.86)$ & 2.19 \\
\hline & 11 Inferior Parietal Lobule & Right & $-0.06 \pm 0.07$ & $>1(0.64)$ & 0.33 & $0.33 \pm 0.07$ & $.0002(22.58)$ & 1.94 & $0.27 \pm 0.07$ & $.009(15.60)$ & 1.61 \\
\hline \multirow[t]{3}{*}{ R Claustrum } & 12 Inferior Frontal Gyrus & Left & $-0.004 \pm 0.05$ & $>1(0.01)$ & 0.03 & $0.29 \pm 0.05$ & $5 \times 10^{-6}(30.03)$ & 2.24 & $0.28 \pm 0.05$ & $8 \times 10^{-6}(29.17)$ & 2.21 \\
\hline & 13 Superior Temporal Gyrus & Left & $0.11 \pm 0.07$ & $>1(2.30)$ & 0.62 & $0.27 \pm 0.07$ & $.019(14.19)$ & 1.54 & $0.38 \pm 0.07$ & $1 \times 10^{-5}(27.91)$ & 2.16 \\
\hline & 14 Inferior Parietal Lobule & Left & $0.01 \pm 0.06$ & $>1(0.01)$ & 0.04 & $0.26 \pm 0.06$ & $.002(18.80)$ & 1.77 & $0.27 \pm 0.06$ & $.001(19.67)$ & 1.81 \\
\hline \multirow[t]{2}{*}{ L Cingulate Gyrus } & 15 Insula & Right & $-0.002 \pm 0.07$ & $>1(0.002)$ & 0.02 & $0.35 \pm 0.07$ & $1 \times 10^{-5}(28.37)$ & 2.17 & $0.34 \pm 0.07$ & $1 \times 10^{-5}(27.97)$ & 2.16 \\
\hline & 16 Medial Frontal Gyrus & Left & $0.04 \pm 0.06$ & $>1(0.51)$ & 0.29 & $0.26 \pm 0.06$ & $.0003(22.06)$ & 1.92 & $0.31 \pm 0.06$ & $7 \times 10^{-6}(29.29)$ & 2.21 \\
\hline \multirow[t]{2}{*}{ L Superior Frontal Gyrus } & 17 Middle Temporal Gyrus & Left & $0.06 \pm 0.07$ & $>1(0.68)$ & 0.34 & $0.25 \pm 0.07$ & $.090(11.26)$ & 1.37 & $0.31 \pm 0.07$ & $.003(17.45)$ & 1.71 \\
\hline & 18 Lentiform Nucleus & Left & $0.10 \pm 0.07$ & $>1(1.71)$ & 0.53 & $0.27 \pm 0.07$ & $.026(13.60)$ & 1.51 & $0.37 \pm 0.07$ & $7 \times 10^{-5}(24.96)$ & 2.04 \\
\hline R Inferior Frontal Gyrus & 19 Inferior Frontal Gyrus & Left & $0.001 \pm 0.05$ & $>1(0.0004)$ & 0.01 & $0.24 \pm 0.05$ & $2 \times 10^{-5}(27.66)$ & 2.15 & $0.24 \pm 0.05$ & $1 \times 10^{-5}(27.87)$ & 2.16 \\
\hline
\end{tabular}

The linear mixed-effects model had a within-participants fixed effect PHASE (Rest-1 vs. Rest-2 vs. Rest-3), a between-participants fixed effect GROUP (VIMS vs. the healthy participant group), the interaction of PHASE $\times$ GROUP, and a random effect of each individual participant. Because there was a PHASE $\times$ GROUP interaction for all 19 brain pairs (Table 3), a post hoc test was conducted on the simple effect of PHASE. For the effect size of the simple effect, Cohen's $d$ was computed. The P values of the post hoc tests were Bonferroni corrected for the number of brain region pairs, participant groups, and comparison repetitions. The numbering of the 19 pairs corresponds to that in Figure 4 and Supplementary Figure 1. 


\begin{tabular}{|c|c|c|c|c|c|c|c|c|c|c|c|c|}
\hline & & & & \multicolumn{3}{|c|}{ Rest-1 vs. Rest-2 } & \multicolumn{3}{|c|}{ Rest-2 vs. Rest-3 } & \multicolumn{3}{|c|}{ Rest-1 vs. Rest-3 } \\
\hline & & & & $\begin{array}{c}\text { Coefficient } \\
\text { Estimate } \pm \text { SE }\end{array}$ & $\begin{array}{l}\text { Test statistics } \\
P \text { value }\left(\chi^{2}\right)\end{array}$ & $\begin{array}{l}\text { Effect size } \\
\text { Cohen's } d\end{array}$ & $\begin{array}{c}\text { Coefficient } \\
\text { Estimate } \pm \mathrm{SE}\end{array}$ & $\begin{array}{l}\text { Test statistics } \\
P \text { value }\left(\chi^{2}\right)\end{array}$ & $\begin{array}{l}\text { Effect size } \\
\text { Cohen's } d\end{array}$ & $\begin{array}{c}\text { Coefficient } \\
\text { Estimate } \pm \mathrm{SE}\end{array}$ & $\begin{array}{l}\text { Test statistics } \\
P \text { value }\left(\chi^{2}\right)\end{array}$ & $\begin{array}{l}\text { Effect size } \\
\text { Cohen's } d\end{array}$ \\
\hline \multirow{3}{*}{ L Thalamus } & & Insula & Right & $-0.01 \pm 0.05$ & $>1(0.07)$ & 0.11 & $-0.06 \pm 0.05$ & $>1(1.25)$ & 0.46 & $-0.07 \pm 0.05$ & $>1(1.92)$ & 0.57 \\
\hline & & Inferior Parietal Lobule & Right & $-0.06 \pm 0.07$ & $>1(0.66)$ & 0.33 & $-0.05 \pm 0.07$ & $>1(0.49)$ & 0.29 & $-0.11 \pm 0.07$ & $>1(2.30)$ & 0.62 \\
\hline & & Declive & Right & $-0.04 \pm 0.06$ & $>1(0.59)$ & 0.31 & $-0.05 \pm 0.06$ & $>1(0.82)$ & 0.37 & $-0.10 \pm 0.06$ & $>1(2.79)$ & 0.68 \\
\hline \multirow{2}{*}{ L Insula } & 4 & Thalamus & Left & $-0.07 \pm 0.07$ & $>1(1.13)$ & 0.43 & $-0.06 \pm 0.07$ & $>1(0.90)$ & 0.39 & $-0.14 \pm 0.07$ & $>1(4.05)$ & 0.82 \\
\hline & 5 & Inferior Parietal Lobule & Right & $-0.11 \pm 0.07$ & $>1(2.30)$ & 0.62 & $-0.04 \pm 0.07$ & $>1(0.24)$ & 0.20 & $-0.07 \pm 0.07$ & $>1(1.05)$ & 0.42 \\
\hline \multirow{3}{*}{ R Insula } & 6 & Thalamus & Left & $-0.02 \pm 0.05$ & $>1(0.13)$ & 0.15 & $-0.04 \pm 0.05$ & $>1(0.88)$ & 0.38 & $-0.06 \pm 0.05$ & $>1(1.70)$ & 0.53 \\
\hline & 7 & Superior Temporal Gyrus & Left & $0.02 \pm 0.06$ & $>1(0.08)$ & 0.11 & $-0.09 \pm 0.06$ & $>1(2.25)$ & 0.61 & $-0.07 \pm 0.06$ & $>1(1.50)$ & 0.50 \\
\hline & 8 & Inferior Temporal Gyrus & Left & $-0.05 \pm 0.05$ & $>1(1.03)$ & 0.41 & $-0.04 \pm 0.05$ & $>1(0.45)$ & 0.27 & $-0.09 \pm 0.05$ & $>1(2.84)$ & 0.69 \\
\hline \multirow{3}{*}{\multicolumn{2}{|c|}{ L Cerebellar Tonsil }} & Parahippocampal Gyrus & Left & $-0.05 \pm 0.07$ & $>1(0.45)$ & 0.27 & $-0.10 \pm 0.07$ & $>1(2.12)$ & 0.59 & $-0.15 \pm 0.07$ & $>1(4.51)$ & 0.87 \\
\hline & & Cingulate Gyrus & Left & $-0.03 \pm 0.05$ & $>1(0.41)$ & 0.26 & $-0.06 \pm 0.05$ & $>1(1.29)$ & 0.46 & $-0.09 \pm 0.05$ & $>1(3.15)$ & 0.72 \\
\hline & & Inferior Parietal Lobule & Right & $0.01 \pm 0.06$ & $>1(0.06)$ & 0.10 & $-0.12 \pm 0.06$ & $>1(4.20)$ & 0.84 & $-0.11 \pm 0.06$ & $>1(3.26)$ & 0.74 \\
\hline \multirow{3}{*}{\multicolumn{2}{|c|}{ R Claustrum }} & Inferior Frontal Gyrus & Left & $-0.08 \pm 0.06$ & $>1(1.63)$ & 0.52 & $-0.03 \pm 0.06$ & $>1(0.25)$ & 0.20 & $-0.11 \pm 0.06$ & $>1(3.14)$ & 0.72 \\
\hline & & Superior Temporal Gyrus & Left & $-0.08 \pm 0.04$ & $>1(4.84)$ & 0.90 & $-0.04 \pm 0.04$ & $>1(1.06)$ & 0.42 & $-0.11 \pm 0.04$ & $.142(10.43)$ & 1.32 \\
\hline & 14 & Inferior Parietal Lobule & Left & $-0.07 \pm 0.05$ & $>1(2.00)$ & 0.58 & $-0.04 \pm 0.05$ & $>1(0.46)$ & 0.27 & $-0.11 \pm 0.05$ & $>1(4.34)$ & 0.85 \\
\hline \multirow{2}{*}{\multicolumn{2}{|c|}{ L Cingulate Gyrus }} & Insula & Right & $-0.06 \pm 0.04$ & $>1(2.55)$ & 0.65 & $-0.06 \pm 0.04$ & $>1(2.69)$ & 0.67 & $-0.12 \pm 0.04$ & $.137(10.49)$ & 1.32 \\
\hline & & Medial Frontal Gyrus & Left & $0.003 \pm 0.03$ & $>1(0.02)$ & 0.06 & $-0.08 \pm 0.03$ & $.313(8.97)$ & 1.22 & $-0.08 \pm 0.03$ & $.502(8.11)$ & 1.16 \\
\hline \multirow{2}{*}{\multicolumn{2}{|c|}{ L Superior Frontal Gyrus }} & Middle Temporal Gyrus & Left & $-0.06 \pm 0.05$ & $>1(1.33)$ & 0.47 & $-0.06 \pm 0.05$ & $>1(0.70)$ & 0.53 & $-0.12 \pm 0.05$ & $>1(6.04)$ & 1.00 \\
\hline & & Lentiform Nucleus & Left & $-0.04 \pm 0.06$ & $>1(0.50)$ & 0.29 & $-0.07 \pm 0.06$ & $>1(1.15)$ & 0.44 & $-0.11 \pm 0.06$ & $>1(3.18)$ & 0.73 \\
\hline R Inferior Frontal Gyrus & 19 & Inferior Frontal Gyrus & Left & $-0.02 \pm 0.05$ & $>1(0.14)$ & 0.15 & $-0.07 \pm 0.05$ & $>1(1.70)$ & 0.53 & $-0.09 \pm 0.05$ & $>1(2.81)$ & 0.68 \\
\hline
\end{tabular}


Click here to access/download;Fig

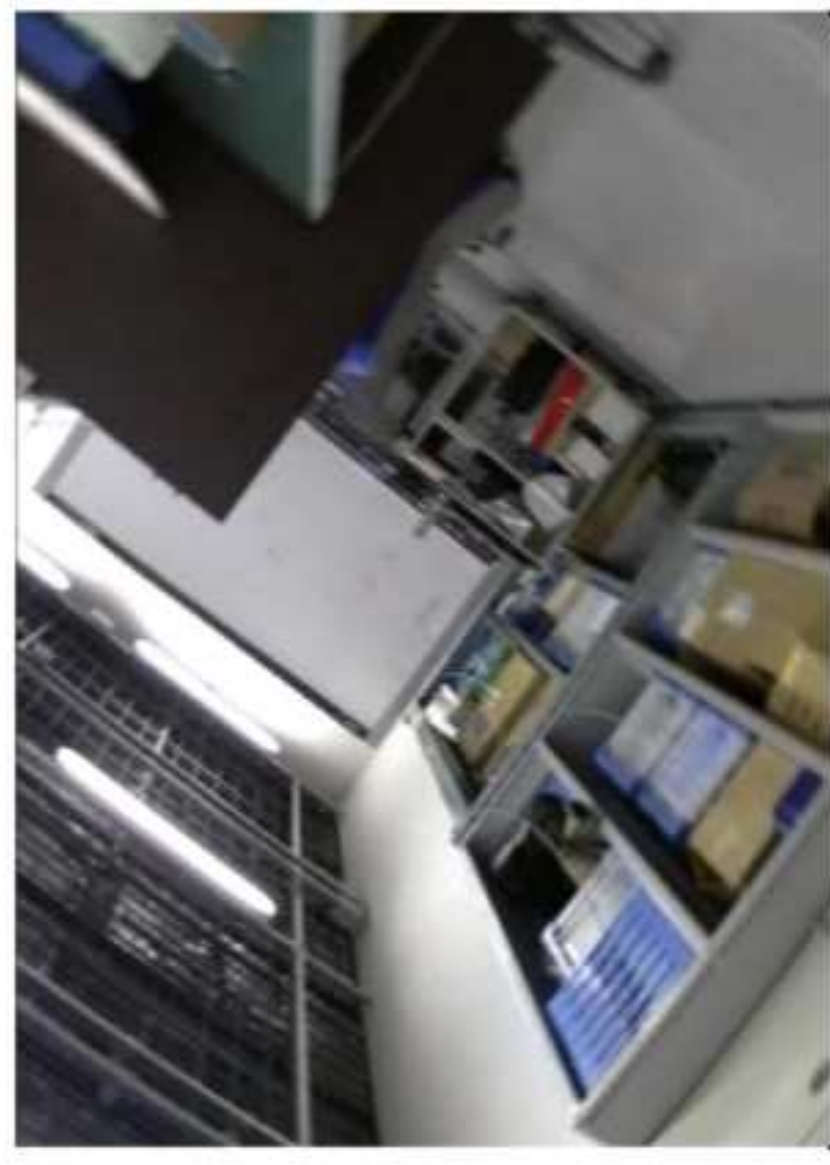

0
0
0

ケ 


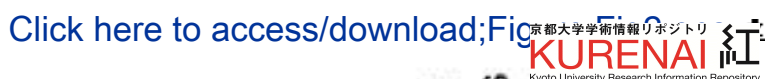

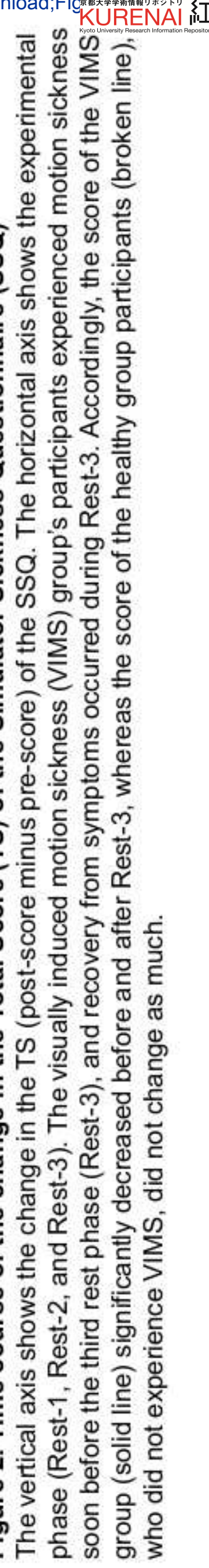

әлоэs 타아 OSS แ! әбиечว

$m$
$\frac{1}{w}$
$\tilde{\alpha}$

N (1)

凹 ठ

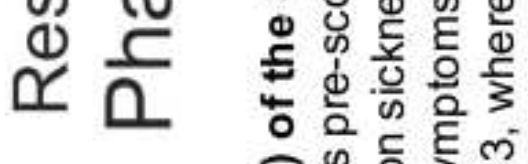

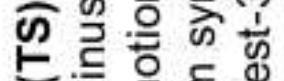

๑ ह ह ठิ

인 원

ஸ

त्ञ

으 으륜

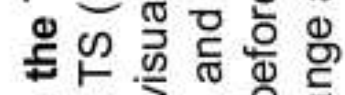

$\subseteq \stackrel{\oplus}{\simeq} \frac{0}{0}$

宁口市 क

잉

동 위는 므

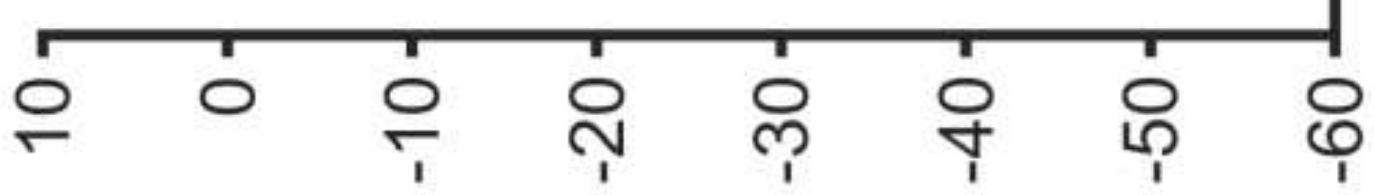

0 도

$\neq 0$ 당

屯ㄷ

0 $\infty$

는

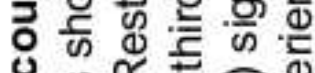

๑

ह ชิ

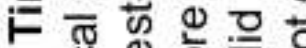

엉

잉

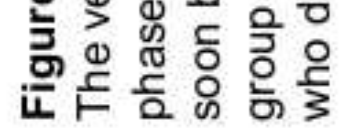


a Left Thalamus

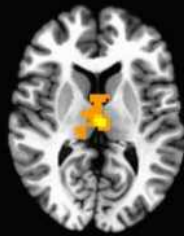

c Left Insula

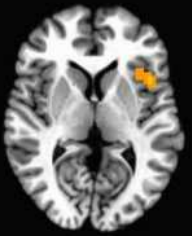

e Cerebellar Tonsil

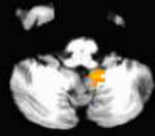

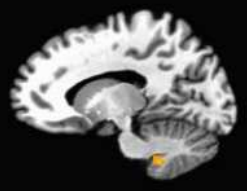

b Anterior Cingulate
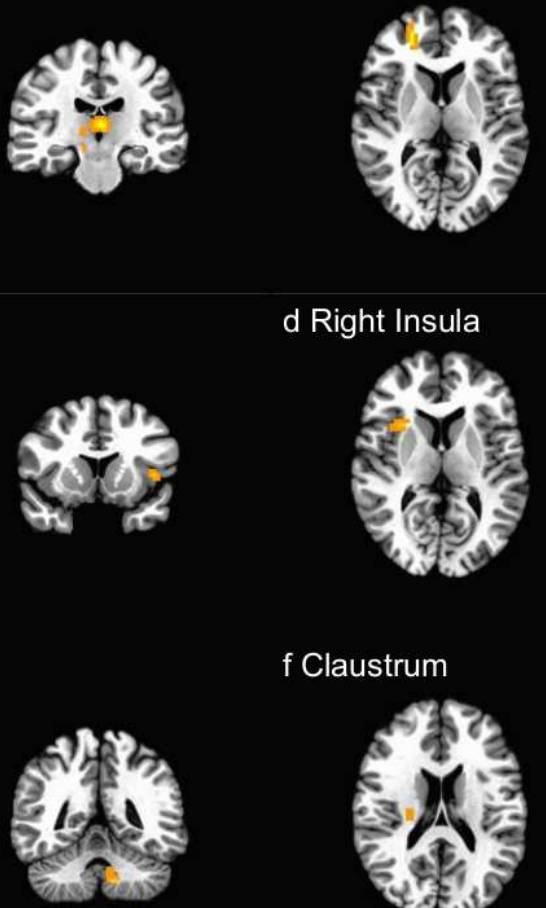

d Right Insula

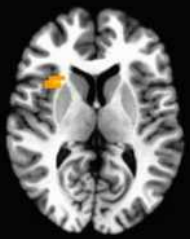

f Claustrum
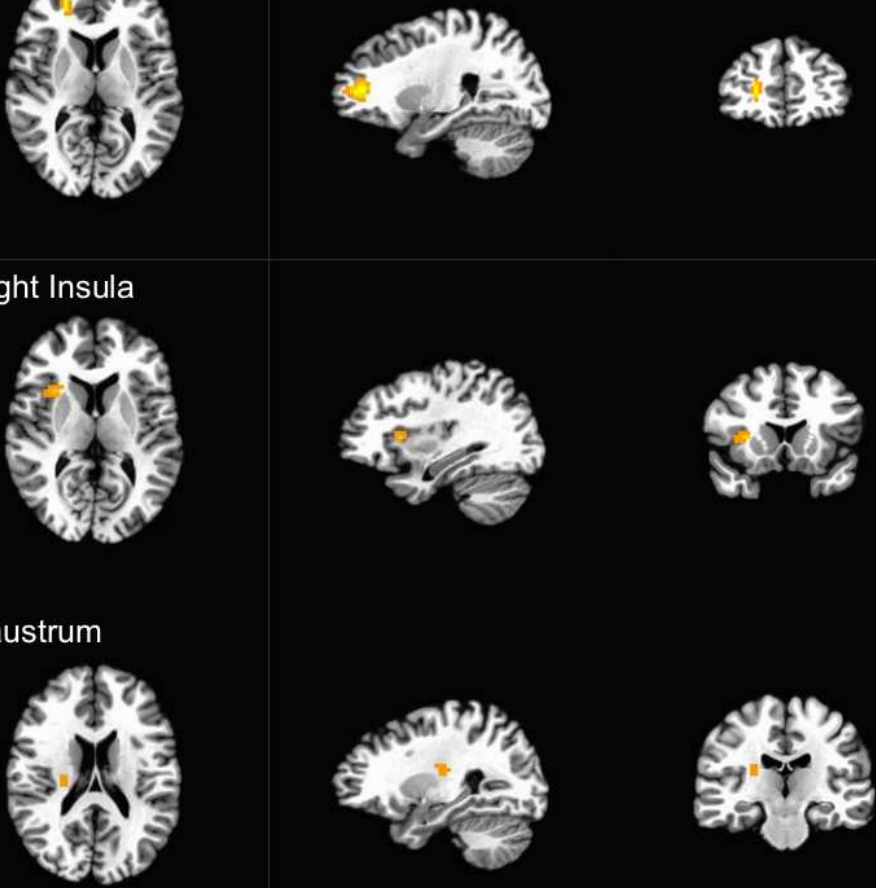

h Cingulate Gyrus
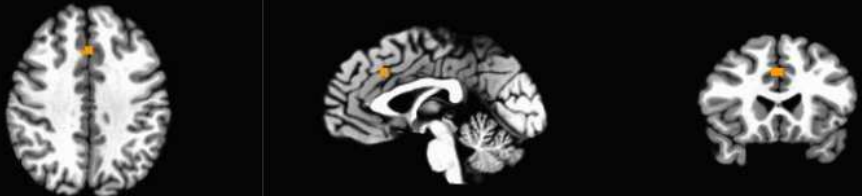

j Superior Frontal Gyrus
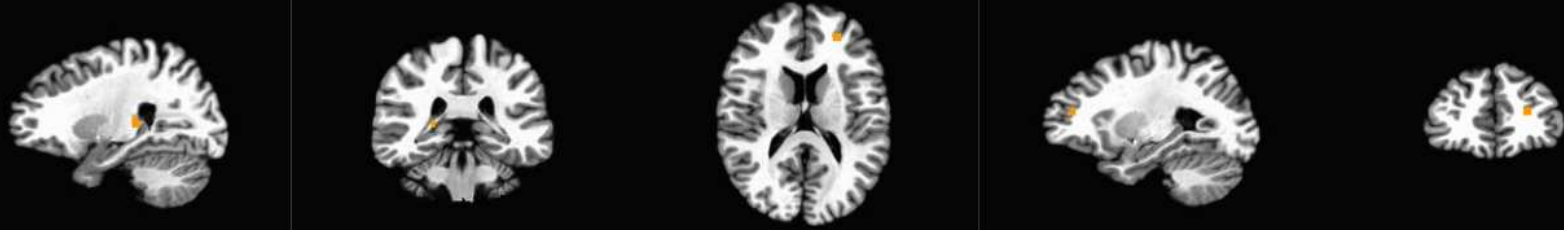

I Inferior Frontal Gyrus
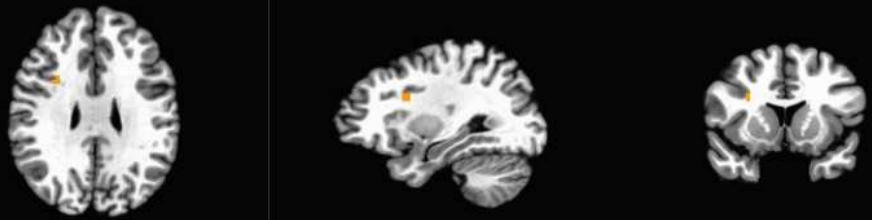

Figure 3. Twelve brain regions whose connectedness significantly increased during the recovery phase from visually induced motion sickness (VIMS)

Each row is as follows: a, left thalamus; b, anterior cingulate; c, left insula; d, right insula; e, cerebellar tonsil; f, claustrum; g, right lingual gyrus; h, cingulate gyrus; i, right thalamus; j, left superior frontal gyrus; k, left lingual gyrus; and I, inferior frontal gyrus. Each column shows, from left to right, axial, sagittal, and coronal brain images. The following thresholds were set: voxel-wise threshold $P<0.0001$, cluster level threshold $\alpha<0.10$. 


\section{a 7. L Superior Temporal Gyrus (seed: R Insula)}

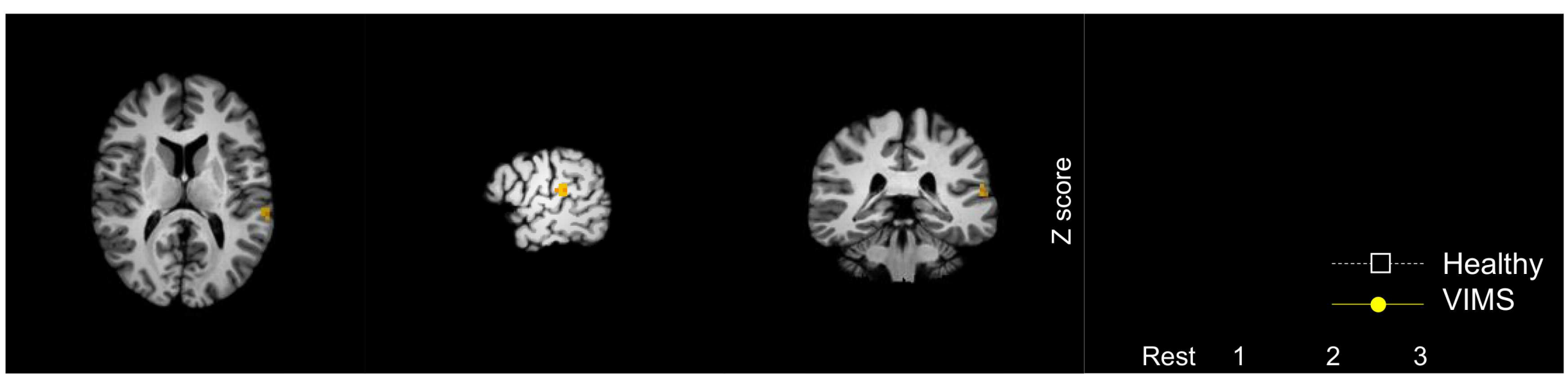

Figure 4. Brain regions whose functional connectivity with the seed regions increased in the recovery phase from visually induced motion sickness (VIMS)

Each column shows, from left to right, axial, sagittal, and coronal brain images, and the change in the functional connectivity ( $Z$ scores averaged within the participants' groups) of each experimental phase, separately for VIMS and healthy groups, respectively (error bars are standard errors). Each panel is the result for the following brain regions (the brain region in brackets is the corresponding seed): a-left superior temporal gyrus (right insula), b-right inferior parietal lobule (right claustrum), c-left superior temporal gyrus (right claustrum), d-left inferior parietal lobule (right claustrum); and e-left lentiform nucleus (left superior frontal gyrus). For this mapping, we used the following thresholds: voxel-wise threshold $P<$ 0.0001 , cluster level threshold $a<0.05$. The numbering of brain region pairs corresponds to that in Tables 3,4 , and 5 . 


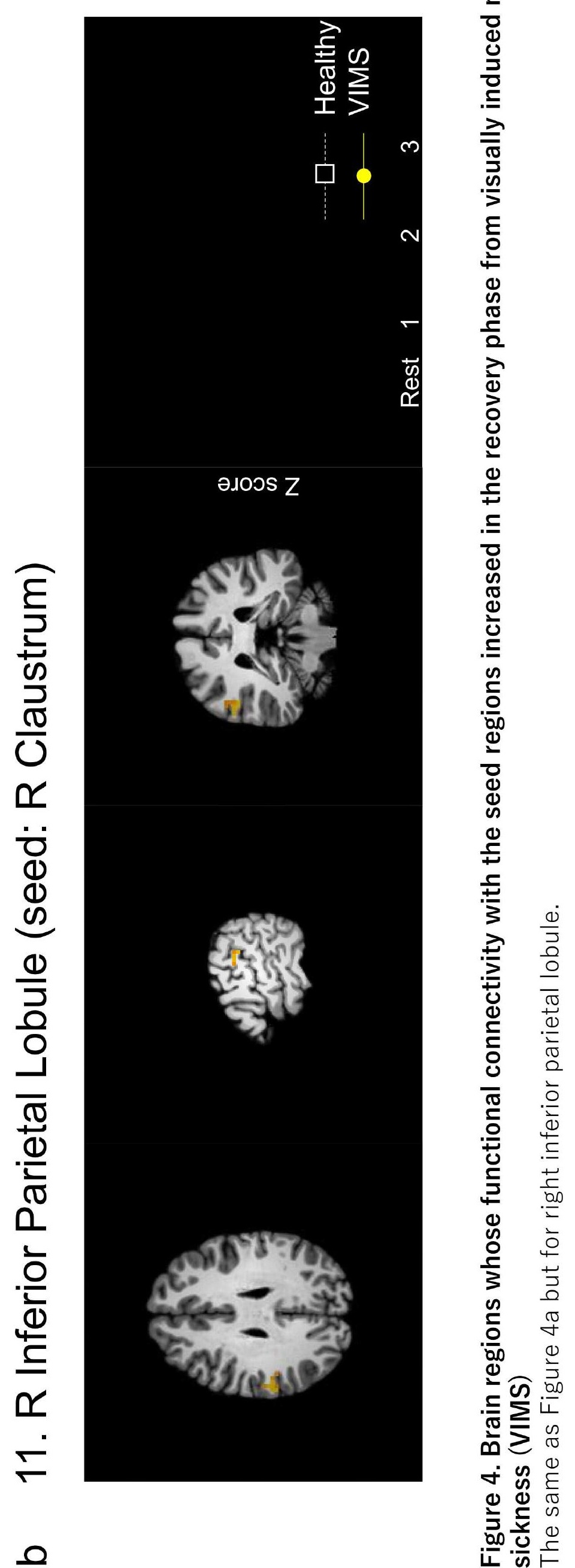




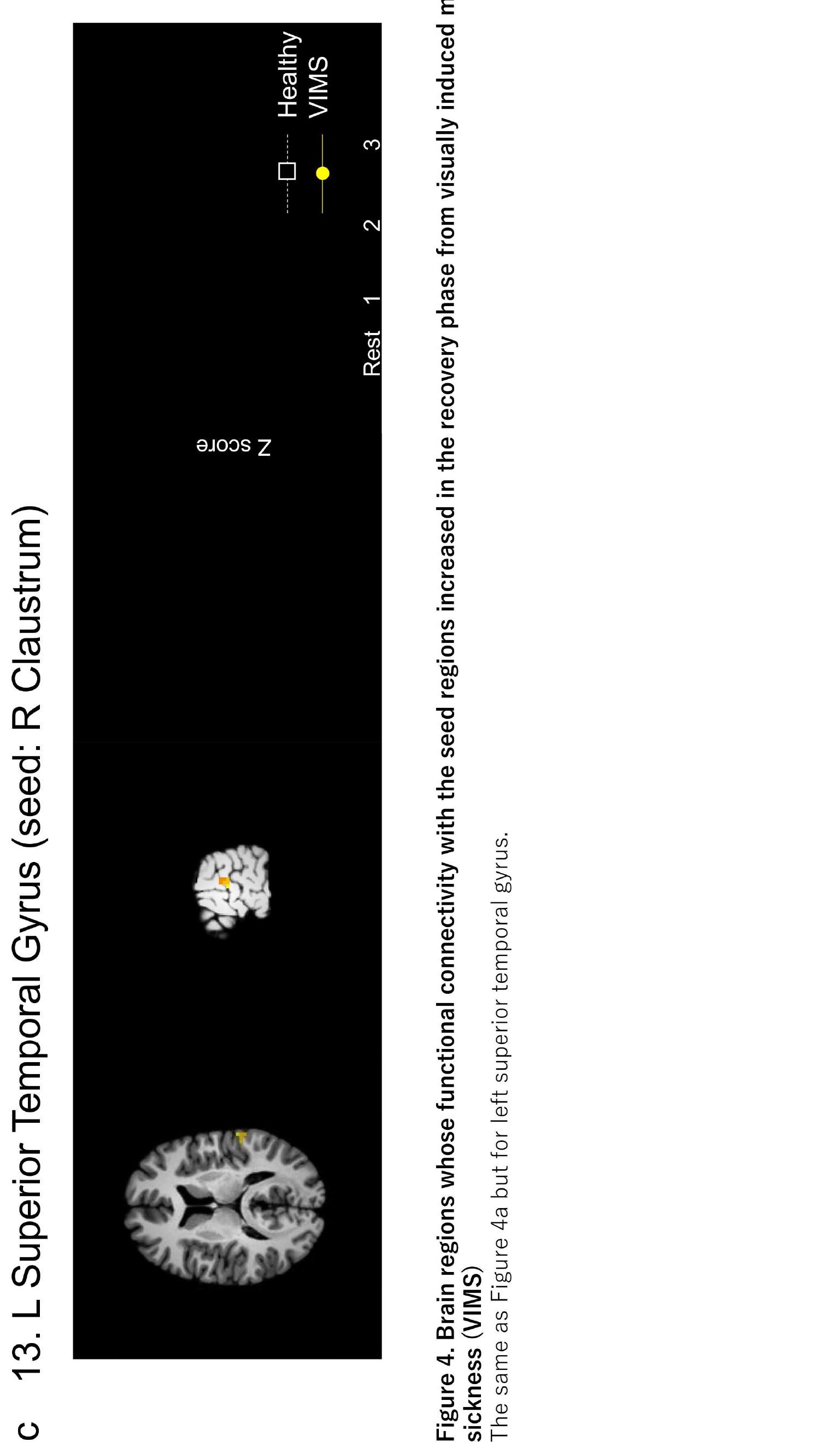




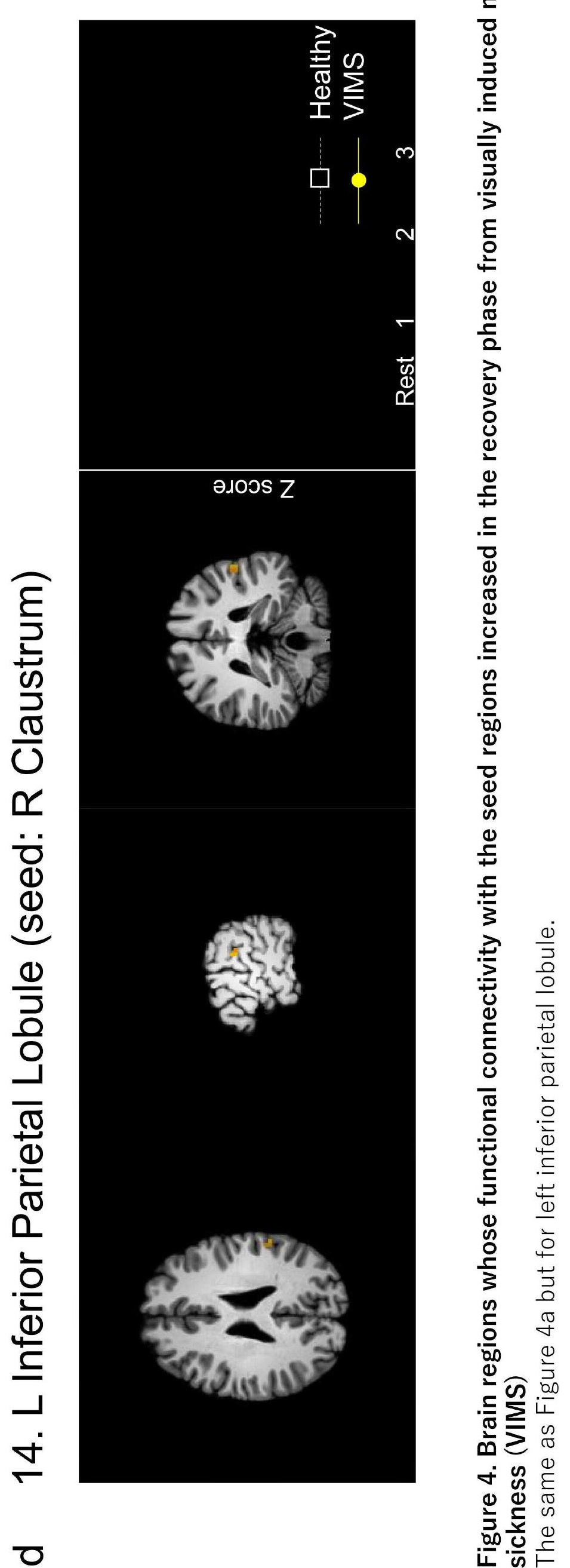




\section{e 18. L Lentiform Nucleus (seed: L Superior Frontal Gyrus)}

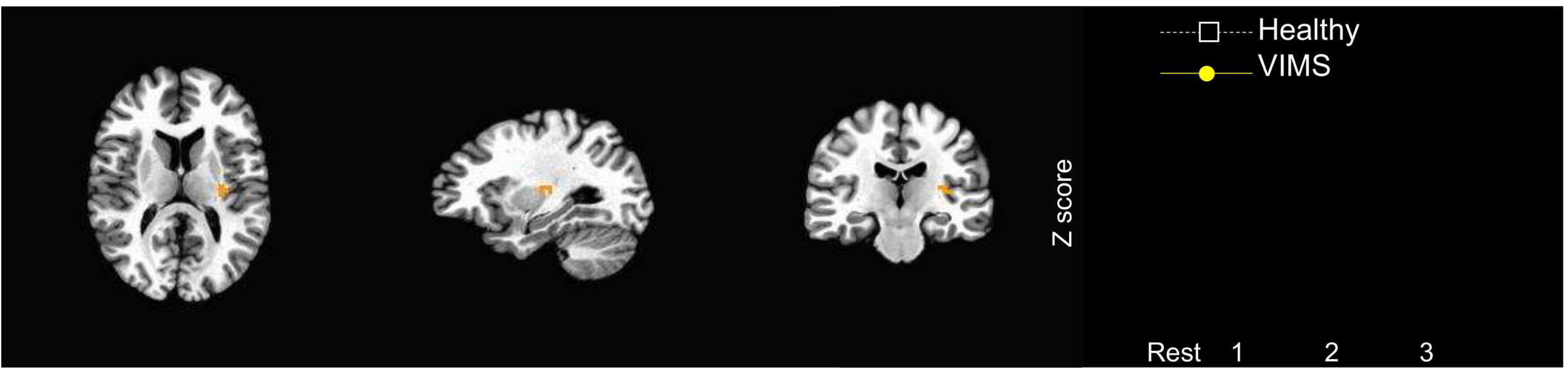

Figure 4. Brain regions whose functional connectivity with the seed regions increased in the recovery phase from visually induced motion sickness (VIMS)

The same as Figure 4a but for left lentiform nucleus. 


\section{$7 \mathrm{R}$ insula - L STG}

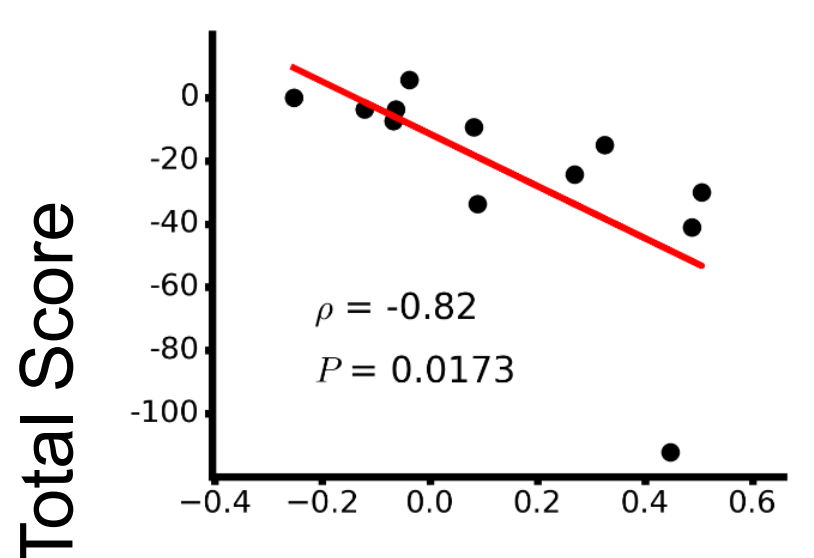

$11 \mathrm{R}$ claustrum - R IPL

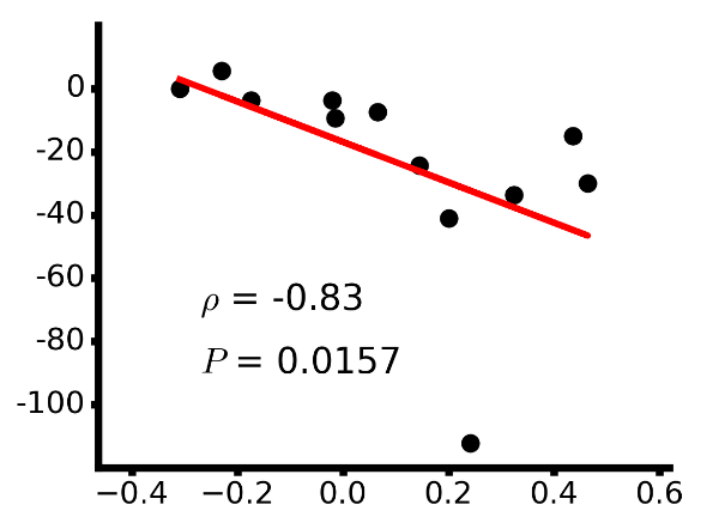

\section{R claustrum - L STG}

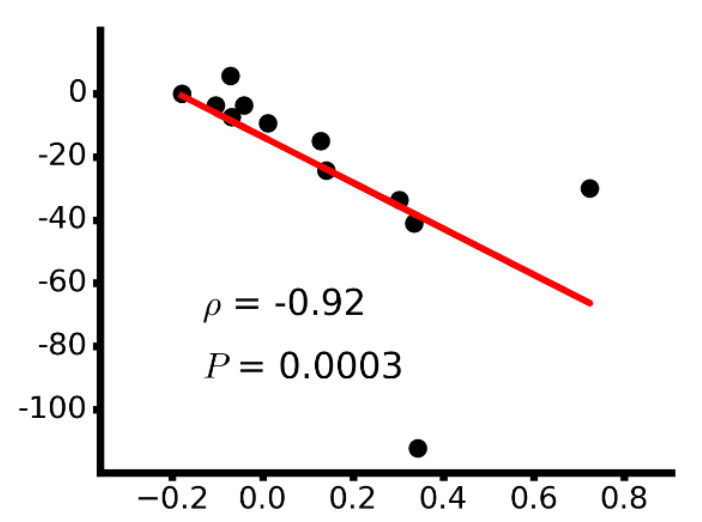

14 R claustrum - L IPL

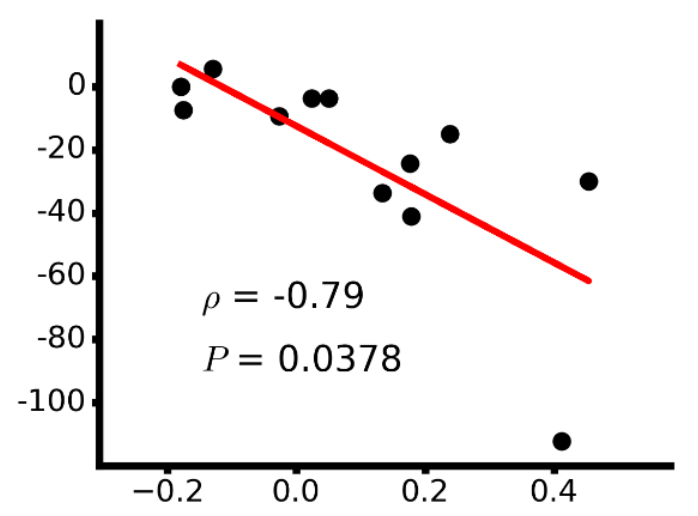

18 L SFG - L lentiform nucleus

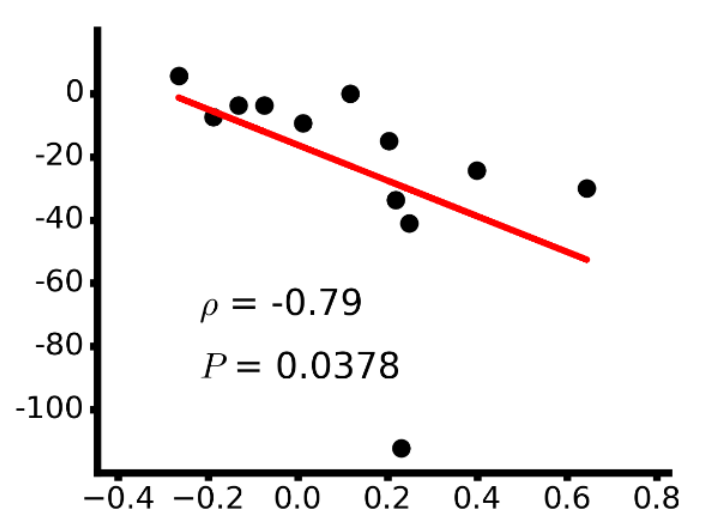

\section{Change in functional connectivity ( $Z$ score)}

Figure 5. Correlation plots between the change in the Total Score (TS) of the Simulator Sickness Questionnaire (SSQ) and the change in functional connectivity

The vertical axis is the change in the TS of the SSQ: Rest-3 minus the average of Rest-1 and Rest-2; the more VIMS symptoms recovered, the more this score decreases. The horizontal axis is the change in functional connectivity: Rest-3 minus the average of Rest-1 and Rest-2. In the graphs, $\rho$ indicates Spearman's rank correlation coefficient, with the corresponding $P$ value shown below. The $P$ values were Bonferroni corrected for multiple comparisons among the 19 brain region pairs. Abbreviations: IPL, inferior parietal lobule; L, left; R, right; SFG, superior frontal gyrus; STG, superior temporal gyrus. 
a VIMS recovery (Rest-3) phase

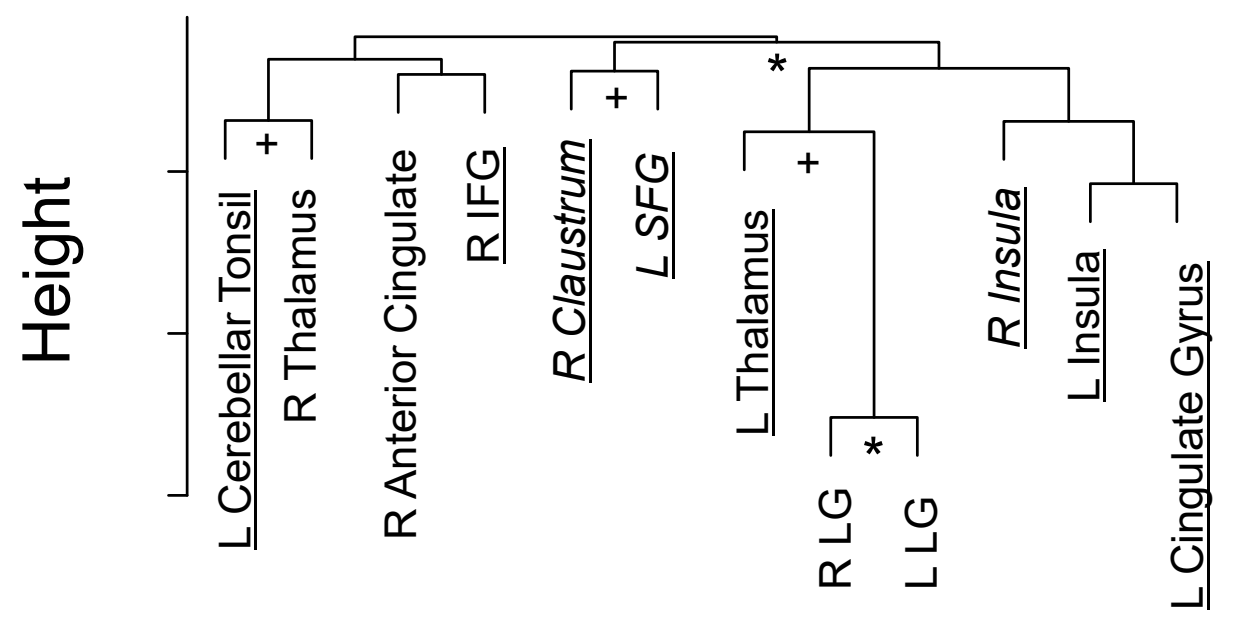

C VIMS control (average of Rest-1 \& -2) phase

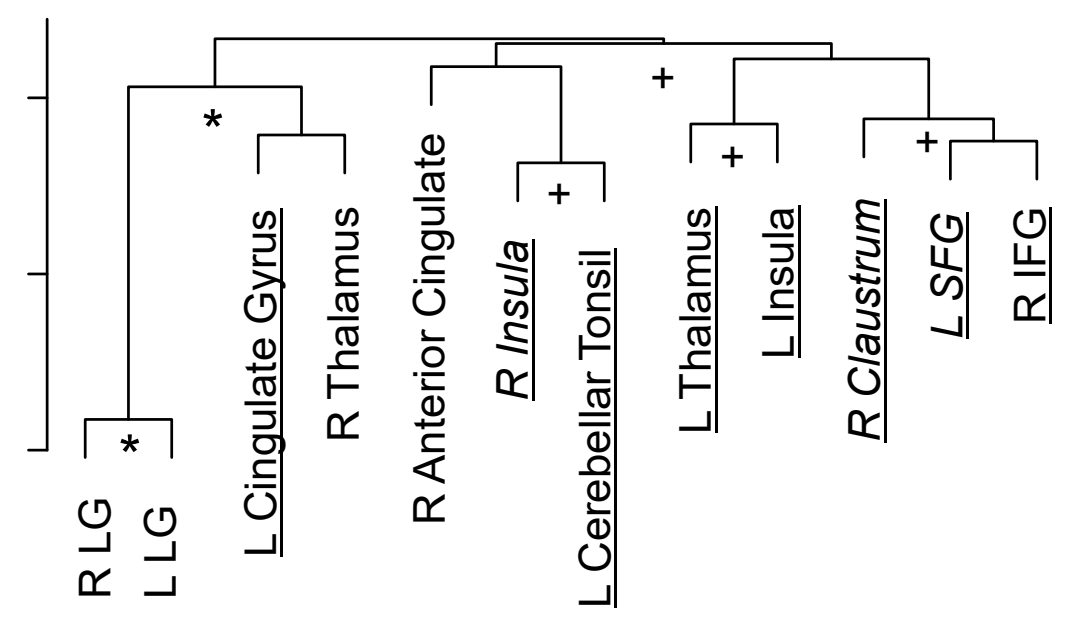

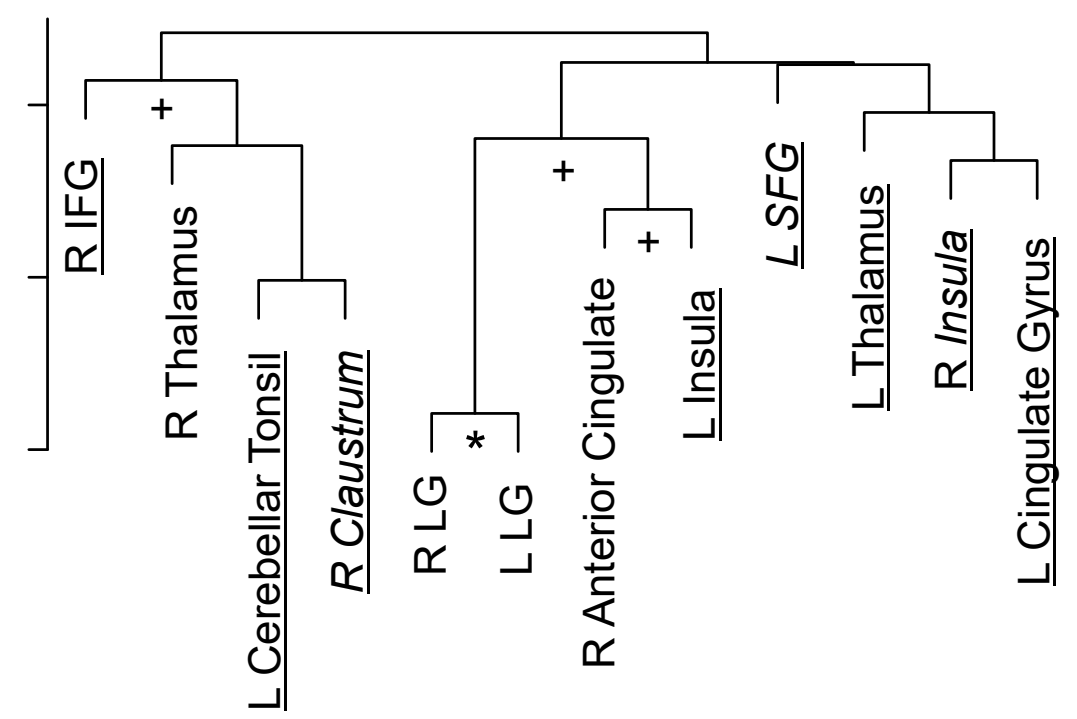

d healthy control (average of Rest-1 \& -2) phase

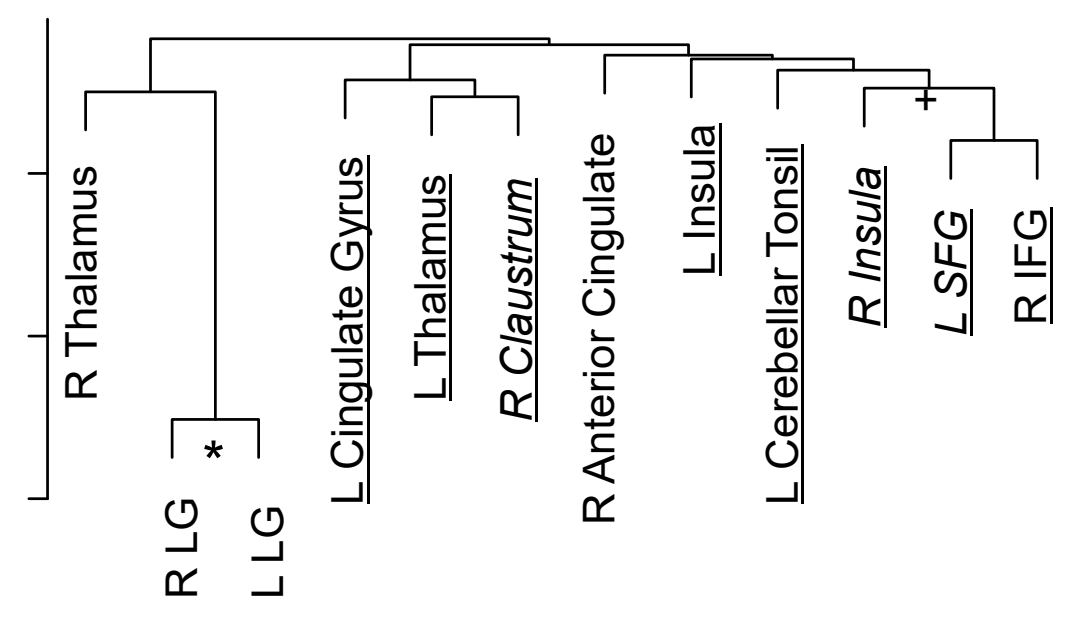

Figure 6. Hierarchical clustering trees of the 12 brain regions showing recovery-selective increases in connectedness

(a) The dendrogram of the VIMS group for the recovery phase (Rest-3), which was derived from the distance matrix (Supplementary Fig. 2a), whose elements indicate 1 minus absolute partial correlation between each pair of the 12 regions. Statistically significant clusters are marked with an asterisk $(P<0.05)$ or plus $(P<0.1)$. Brain regions showing recovery-selective increases in the ROI-based functional connectivity and correlations with SSQ are underlined and in italics, respectively. (b) The same as (a) but for the healthy group. (c) The same as (a) but for the control phase (the average of Rest-1 and Rest-2). (d) The same as (c) but for the healthy group. Abbreviations: L, left; R, right; LG, lingual gyrus; SFG, superior frontal gyrus; IFG, inferior frontal gyrus. 


\title{
+Supplementary Materials
}

\section{Resting-state functional connectivity predicts recovery from visually induced motion sickness}

\author{
Jungo Miyazaki ${ }^{1}$, Hiroki Yamamoto ${ }^{2 *}$, Yoshikatsu Ichimura ${ }^{3}$, Hiroyuki \\ Yamashiro $^{4}$, Tetsuya Yamamoto ${ }^{5}$, Tomokazu Murase ${ }^{6}$, Masahiro Umeda ${ }^{7}$, Toshihiro \\ Higuchi $^{6}$ \\ ${ }^{1}$ Corporate R\&D, Kyocera Corp., Kanagawa, Japan \\ ${ }^{2}$ Graduate School of Human and Environmental Studies, Kyoto University, Kyoto, \\ Japan \\ ${ }^{3}$ Corporate R\&D, Canon Inc., Tokyo, Japan \\ ${ }^{4}$ Department of Medical Engineering, Aino University, Osaka, Japan \\ ${ }^{5}$ Department of System Neuroscience, National Institute for Physiological \\ Sciences, Aichi, Japan \\ Departments of ${ }^{6}$ Neurosurgery and ${ }^{7}$ Medical Informatics, Meiji University of \\ Oriental Medicine, Kyoto, Japan
}

*Correspondence to: Dr. Hiroki Yamamoto

Graduate School of Human and Environmental Studies, Kyoto University Yoshida Nihonmatsu-cho, Sakyo-ku, Kyoto 606-8501, Japan

E-mail: yamamoto@cv.jinkan.kyoto-u.ac.jp

Tel: +81-75-753-2978; Fax: +81-75-753-6574 


\section{Statistical assessment of the effects of head motion on functional connectivity}

To clarify whether head motion affects functional connectivity, we statistically assessed the effect of head movement on functional connectivity. As a measure of head movement, the framewise displacement (FD) (Power et al. 2012) was computed. Then, the maximum value of FD during each rest phase was derived, and the effect of this maximum FD on the functional connectivity of 19 brain region pairs (listed in Tables 3-5) was tested with a linear mixed-effects model analysis. The linear mixed-effects model had fixed effects of the maximum FD (hereafter "FD") in addition to PHASE (Rest-1, -2, and -3) and GROUP (VIMS and healthy group) and three interactions of FD $\times$ PHASE, FD $\times$ GROUP, and PHASE $\times$ GROUP, as well as a random intercept for participants. A standard model selection technique was conducted using the step function in the lmerTest package (Kuznetsova et al. 2017) of $\mathrm{R}$ software. The step function statistically tests fixed effects based on the Satterthwaite method and removes ineffective terms from the model.

All 19 brain regions again showed a significant PHASE $\times$ GROUP interaction, confirming our results in the main text. The results for the effect of FD are shown in Supplementary Table 1, in which $P$ values were not adjusted. For 15 brain region pairs among the 19 pairs, three head motion-related terms (that is the fixed effect of FD and the interactions of FD $\times$ PHASE and FD $\times$ GROUP) were not statistically significant, suggesting no effect of head motion on functional connectivity. A statistically significant FD $\times$ GROUP interaction was obtained for the remaining four pairs: the right claustrum-right inferior parietal lobule, right claustrum-left superior temporal gyrus, right claustrum-left inferior parietal lobule, and left superior frontal gyrus-left medial frontal gyrus. The PHASE $\times$ GROUP interaction was again statistically significant, even when the FD $\times$ GROUP interaction term and the simple effect of FD were included in the model as covariants. The post hoc interaction analyses essentially showed the same results for the VIMS group as those obtained with the original model without the covariants. For the healthy group, a slightly different result was obtained. There were simple effects of PHASE not found by the original model for two of the four pairs: the right claustrum-right inferior parietal lobule and the left superior frontal gyrus-left medial frontal gyrus. For these pairs, recovery-selective decreases in functional connectivity were shown for Rest-3 compared with Rest-2.

\section{Extraction of independent brain networks with dictionary learning}

To complement our main results, we performed a separate network analysis that extracted statistically independent resting networks related to the recovery from VIMS. A dictionary learning technique (Mensch et al. 2016) was applied for the fMRI data of the individuals in the VIMS group, using the nilearn module of Python (https://nilearn.github.io/). First, after preprocessing of the fMRI data as described in the main text, the data during the recovery phase were subjected to dictionary learning (DictLearn and RegionExtractor methods with the number of components $=15$ and 
minimum region size $=100$ voxels), revealing 22 sparse regions of brain activities. Second, for each pair of the 22 brain regions, cross-correlation coefficients of fMRI time-series were computed, leading to a $22 \times 22$ matrix. Finally, this matrix was subjected to a connectome analysis using the plot_connectome function (edge threshold $=90 \%$ ) in nilearn.

The result is shown in Supplementary Figure 3. Multiple brain regions critical for VIMS dynamics were found. The connectome map (Supplementary Figure 3b) shows visual processing regions, including the middle temporal gyrus and lingual gyrus, which were also found in the connectedness/functional connectivity approach used in the main text. These visual regions have been reported to have connectivity changes in the evolutional phase of VIMS (Miyazaki et al. 2015; Toschi et al. 2017). In addition, the cingulate region, which has been indicated to play key roles in the interoceptive process, such as self-awareness to one's own unpleasant bodily state, during/after VIMS, was detected in this analysis as well as that of the main text (Tables 2-5). Interestingly, the parahippocampal gyrus was again extracted in this analysis. The parahippocampal region is a core area for spatial memory processing (Epstein et al. 1998; Epstein, 2008), and memory processing has been suggested to be involved in an adaptation to motion sickness including VIMS (Reason \& Brand 1975; Reason 1978; Oman 1990). This result thus suggests that such an adaptive process might underlie the recovery from VIMS. Altogether, this analysis corroborates our results reported in the main text.

\section{References}

Epstein, R. \& Kanwisher, N. (1998) A cortical representation of the local visual environment. Nature, 392, 598.

Epstein, R.A. (2008) Parahippocampal and retrosplenial contributions to human spatial navigation. Trends in cognitive sciences, 12, 388-396.

Mensch, A., Mairal, J., Thirion, B. \& Varoquaux, G. (Year) Dictionary learning for massive matrix factorization. City. p. 1737-1746.

Oman, C.M. (1990) Motion sickness: a synthesis and evaluation of the sensory conflict theory. Canadian journal of physiology and pharmacology, 68, 294-303.

Power, J.D., Barnes, K.A., Snyder, A.Z., Schlaggar, B.L. \& Petersen, S.E. (2012) Spurious but systematic correlations in functional connectivity MRI networks arise from subject motion. Neuroimage, 59, 2142-2154.

Reason, J.T. (1978) Motion sickness adaptation: a neural mismatch model. Journal of the Royal Society of Medicine, 71, 819-829.

Reason, J.T. \& Brand, J.J. (1975) Motion sickness. Academic press.

Toschi, N., Kim, J., Sclocco, R., Duggento, A., Barbieri, R., Kuo, B. \& Napadow, V. (2017) Motion sickness increases functional connectivity between visual motion and nausea-associated brain regions. Autonomic Neuroscience, 202, 108-113. 


\begin{tabular}{|c|c|c|c|c|c|c|}
\hline \multirow{2}{*}{ Seed region } & \multirow{2}{*}{\multicolumn{2}{|c|}{ Paired region }} & \multirow{2}{*}{ Hemispgere } & \multicolumn{3}{|c|}{$\begin{array}{c}\text { Test statistics } \\
P \text { value }(F \text { value })\end{array}$} \\
\hline & & & & FD & FD $\times$ GROUP & $\mathrm{FD} \times \mathrm{PHASE}$ \\
\hline \multirow{3}{*}{ L Thalamus } & & Insula & Right & $.739(.113)$ & $.501(.722)$ & $.578(.318)$ \\
\hline & 2 & Inferior Parietal Lobule & Right & $.359(.871)$ & $.739(.113)$ & $.819(.203)$ \\
\hline & & Declive & Right & $.963(.002)$ & $.760(.280)$ & $.896(.017)$ \\
\hline \multirow{2}{*}{ L Insula } & 4 & Thalamus & Left & $.929(.008)$ & $.241(1.44)$ & $.888(.120)$ \\
\hline & 5 & Inferior Parietal Lobule & Right & $.438(.621)$ & $.890(.020)$ & $.941(.061)$ \\
\hline \multirow{3}{*}{ R Insula } & 6 & Thalamus & Left & $.671(.185)$ & $.754(.100)$ & $.622(.490)$ \\
\hline & 7 & Superior Temporal Gyrus & Left & $.459(.563)$ & $.191(1.80)$ & $.396(.974)$ \\
\hline & 8 & Inferior Temporal Gyrus & Left & $.312(1.06)$ & $.082(.3 .26)$ & $.661(.424)$ \\
\hline \multirow[t]{3}{*}{ L Cerebellar Tonsil } & 9 & Parahippocampal Gyrus & Left & $.248(1.40)$ & $.086(2.85)$ & $.285(1.19)$ \\
\hline & & Cingulate Gyrus & Left & $.151(2.19)$ & $.362(.860)$ & $.624(.485)$ \\
\hline & 11 & Inferior Parietal Lobule & Right & - & $.008(8.22)$ & $.596(.533)$ \\
\hline \multirow[t]{3}{*}{ R Claustrum } & 12 & Inferior Frontal Gyrus & Left & $.633(.233)$ & $.162(2.04)$ & $.550(.368)$ \\
\hline & 13 & Superior Temporal Gyrus & Left & - & $.030(5.26)$ & $.793(.235)$ \\
\hline & 14 & Inferior Parietal Lobule & Left & - & $.046(4.37)$ & $.733(.316)$ \\
\hline \multirow[t]{2}{*}{ L Cingulate Gyrus } & 15 & Insula & Right & $.542(.381)$ & $.671(4.08)$ & $.973(.001)$ \\
\hline & 16 & Medial Frontal Gyrus & Left & - & $.022(6.11)$ & $.367(1.07)$ \\
\hline \multirow[t]{2}{*}{ L Superior Frontal Gyrus } & 17 & Middle Temporal Gyrus & Left & $.88(3.28)$ & $.525(.420)$ & $.744(.301)$ \\
\hline & & Lentiform Nucleus & Left & $.130(2.47)$ & $.227(1.54)$ & $.518(.683)$ \\
\hline R Inferior Frontal Gyrus & 19 & Inferior Frontal Gyrus & Left & $.485(.502)$ & $.364(.857)$ & $.418(.921)$ \\
\hline
\end{tabular}

The effect of head motion (maximum framewise displacement [FD]) on functional connectivity was statistically assessed by using a linear mixed-effects model, in which a within-participants fixed effect of FD and interactions of FD $\times$ PHASE and FD $\times$ GROUP were included, in addition to the fixed effects of PHASE, GROUP, and PHASE $\times$ GROUP and a random effect of each individual participant. If the head movement-related interactions (i.e., FD $\times$ PHASE and FD $\times$ GROUP) were statistically significant, the statistics for FD were omitted because it was difficult to determine the effect. 


\section{a $1 . R$ Insula (seed: $R$ Thalamus)}

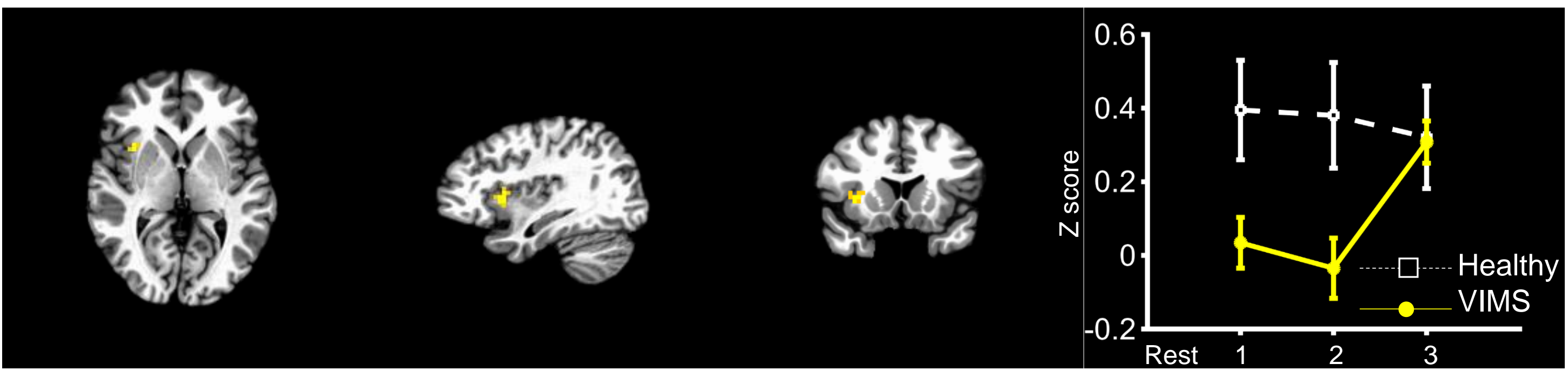

Supplementary Figure 1. Brain regions whose functional connectivity with the seed regions increased in the recovery phase from visually induced motion sickness (VIMS)

Each column shows, from left to right, axial, sagittal, and coronal brain images, and the change in functional connectivity (Z scores averaged within the participants' groups) of each experimental phase, separately for VIMS and healthy groups, respectively (error bars are standard errors). Each panel is the result for the following brain regions (the brain region in brackets is the corresponding seed): a-right insula (right thalamus), bright inferior parietal lobule (right thalamus), c-right declive (right thalamus), d-left thalamus (left insula), e-right inferior parietal lobule (left insula), f-left thalamus (right insula), g-left inferior temporal gyrus (right insula), h-left parahippocampal gyrus (left cerebellar tonsil), i-left cingulate gyrus (right claustrum), j-left inferior frontal gyrus (right claustrum), k-right insula (left cingulate gyrus), I-left medial frontal gyrus (left superior frontal gyrus), m-left middle temporal gyrus (left superior frontal gyrus); and n-left inferior frontal gyrus (right inferior frontal gyrus). For this mapping, we used the following thresholds: voxel-wise threshold $P<0.0001$, cluster level threshold $\alpha<0.05$. The numbering of brain region pairs corresponds to that in Tables 3, 4, and 5. 
b 2. R Inferior Parietal Lobule (seed: R Thalamus)

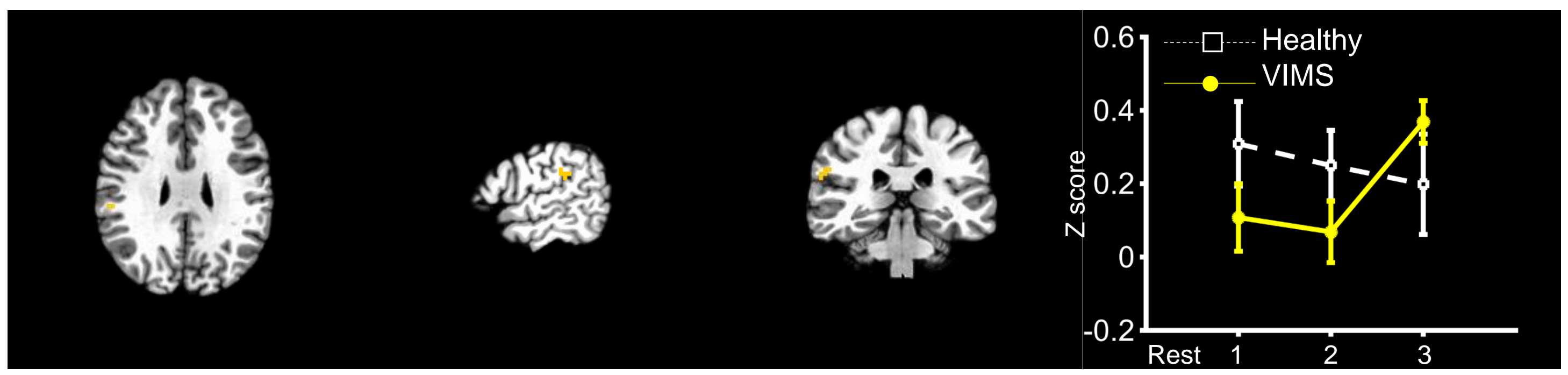

Supplementary Figure 1. Brain regions whose functional connectivity with the seed regions increased in the recovery phase from visually induced motion sickness (VIMS)

The same as Supplementary Figure 1a but for right inferior parietal lobule. 


\section{c $\quad$ 3. $R$ Declive (seed: $R$ Thalamus)}

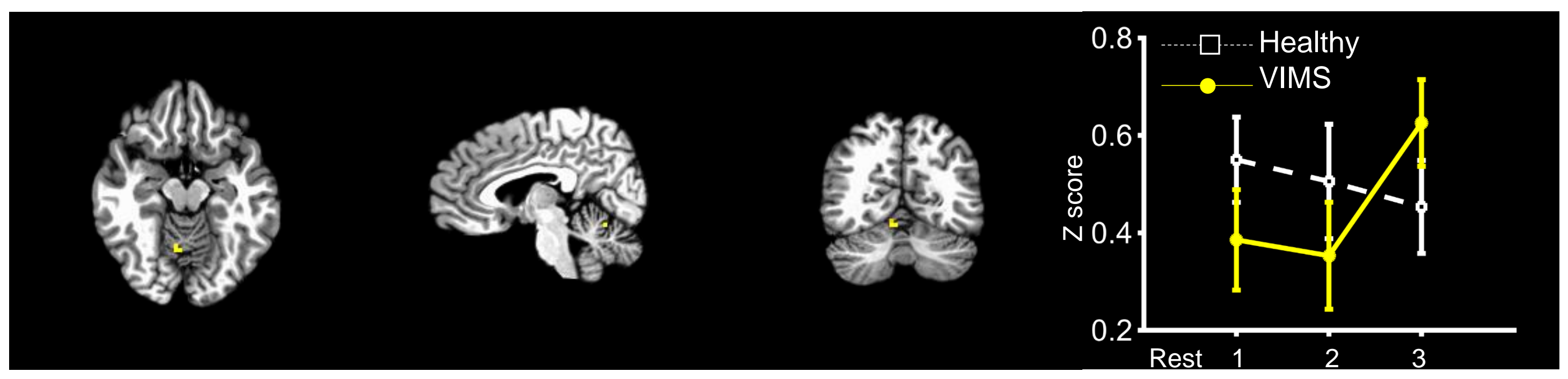

Supplementary Figure 1. Brain regions whose functional connectivity with the seed regions increased in the recovery phase from visually induced motion sickness (VIMS)

The same as Supplementary Figure 1a but for right declive. 
d 4. L Thalamus (seed: L Insula)

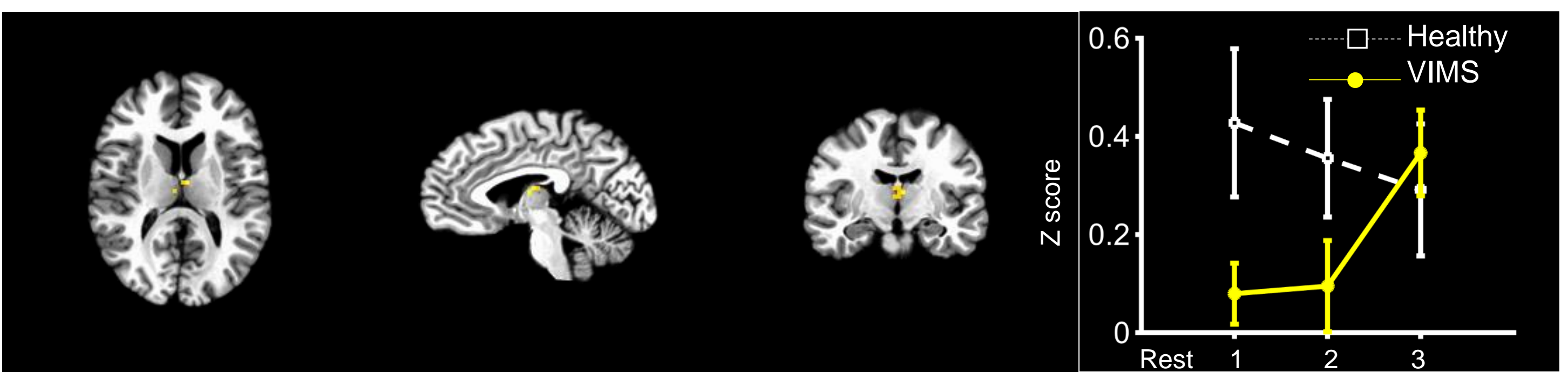

Supplementary Figure 1. Brain regions whose functional connectivity with the seed regions increased in the recovery phase from visually induced motion sickness (VIMS)

The same as Supplementary Figure 1a but for left thalamus. 


\section{e $\quad$ 5. $R$ Inferior Parietal Lobule (seed: L Insula)}

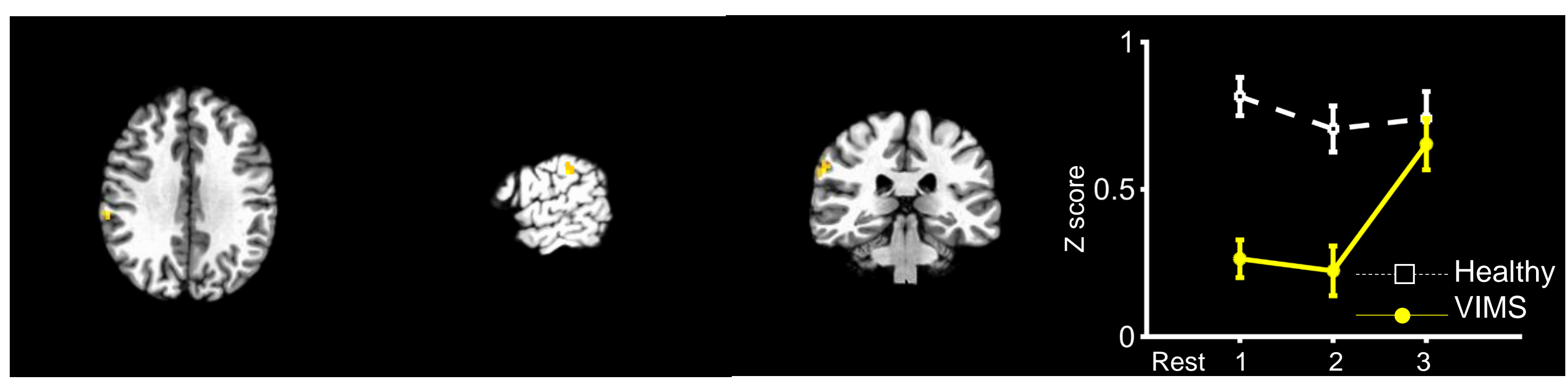

Supplementary Figure 1. Brain regions whose functional connectivity with the seed regions increased in the recovery phase from visually induced motion sickness (VIMS)

The same as Supplementary Figure 1a but for right inferior parietal lobule. 
f $\quad$ 6. L Thalamus (seed: R Insula)

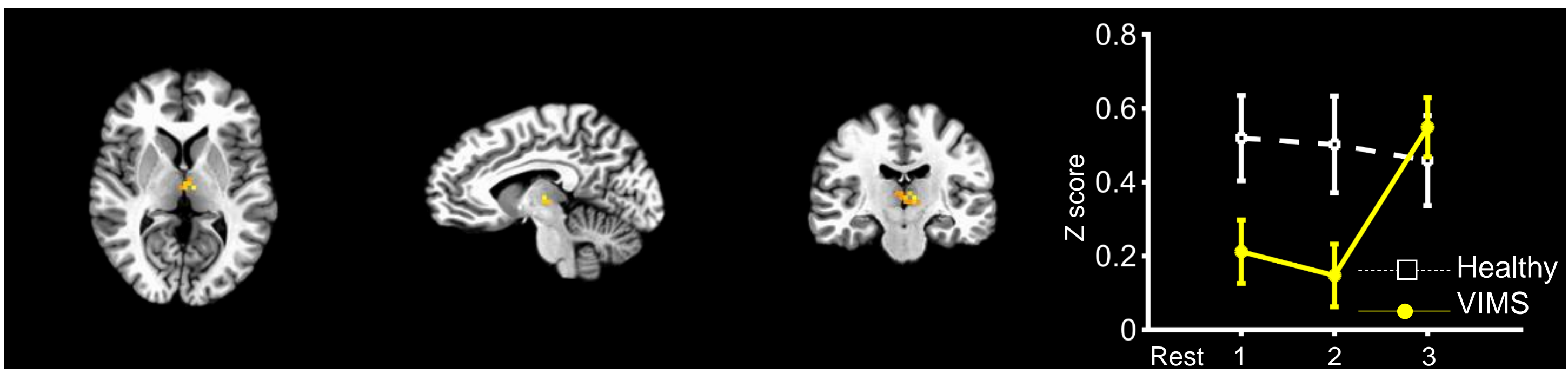

Supplementary Figure 1. Brain regions whose functional connectivity with the seed regions increased in the recovery phase from visually induced motion sickness (VIMS)

The same as Supplementary Figure 1a but for left thalamus. 


\section{g $\quad$ 8. L Inferior Temporal Gyrus (seed: R Insula)}

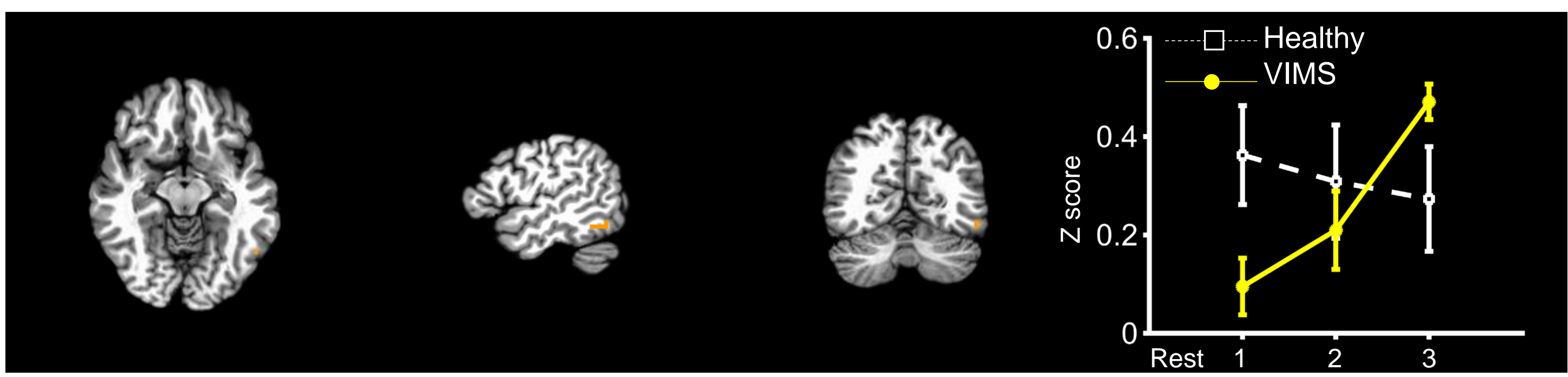

Supplementary Figure 1. Brain regions whose functional connectivity with the seed regions increased in the recovery phase from visually induced motion sickness (VIMS)

The same as Supplementary Figure 1a but for left inferior temporal gyrus. 


\section{h $\quad$ 9. L Parahippocampal Gyrus (seed: L Cerebellar Tonsil)}

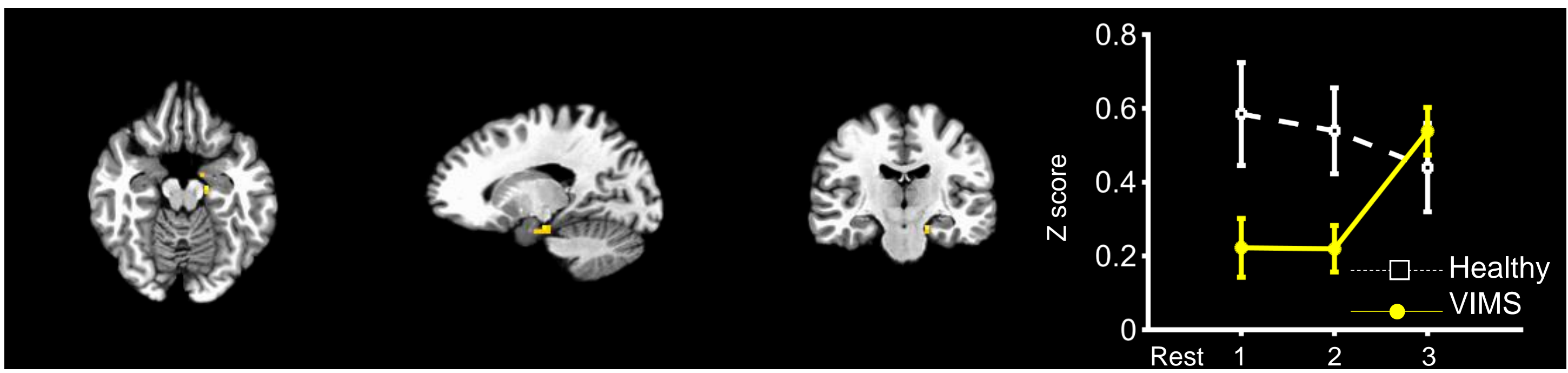

Supplementary Figure 1. Brain regions whose functional connectivity with the seed regions increased in the recovery phase from visually induced motion sickness (VIMS)

The same as Supplementary Figure 1a but for left parahippocampal gyrus. 


\section{L Cingulate Gyrus (seed: R Claustrum)}

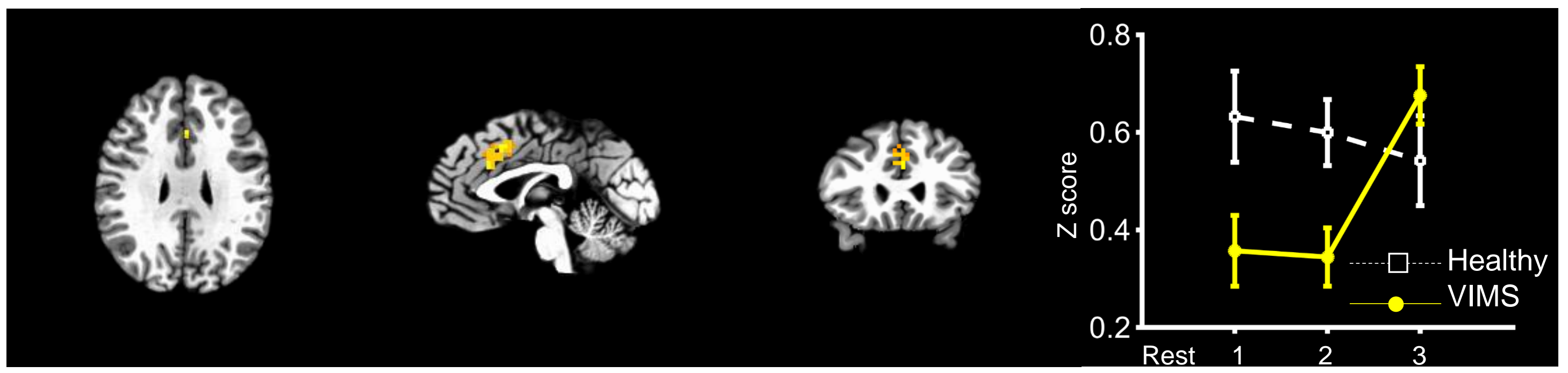

Supplementary Figure 1. Brain regions whose functional connectivity with the seed regions increased in the recovery phase from visually induced motion sickness (VIMS)

The same as Supplementary Figure 1a but for left cingulate gyrus. 
j 12. L Inferior Frontal Gyrus (seed: R Claustrum)

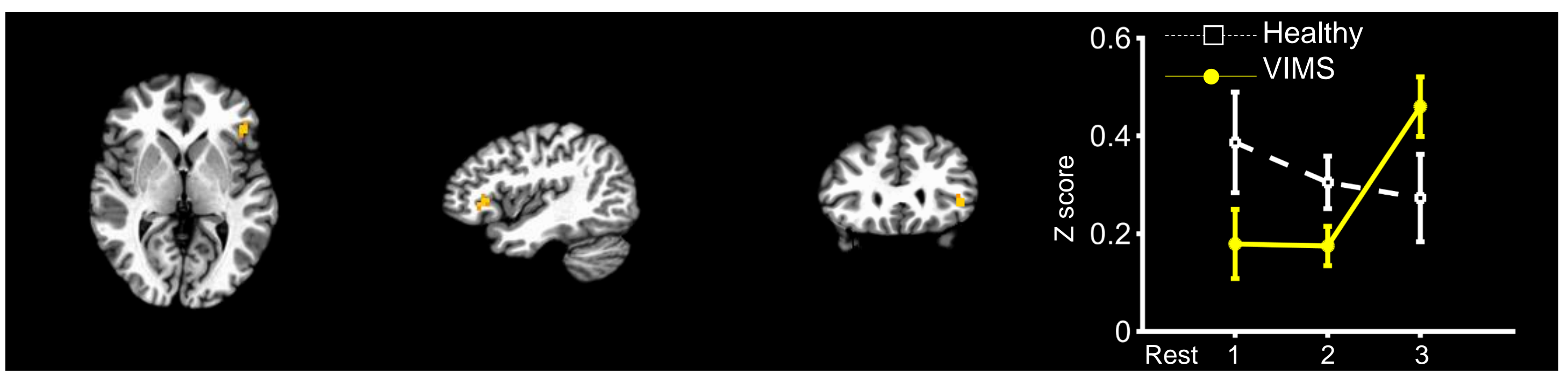

Supplementary Figure 1. Brain regions whose functional connectivity with the seed regions increased in the recovery phase from visually induced motion sickness (VIMS)

The same as Supplementary Figure 1a but for left inferior frontal gyrus. 
k 15. R Insula (seed: L Cingulate Gyrus)

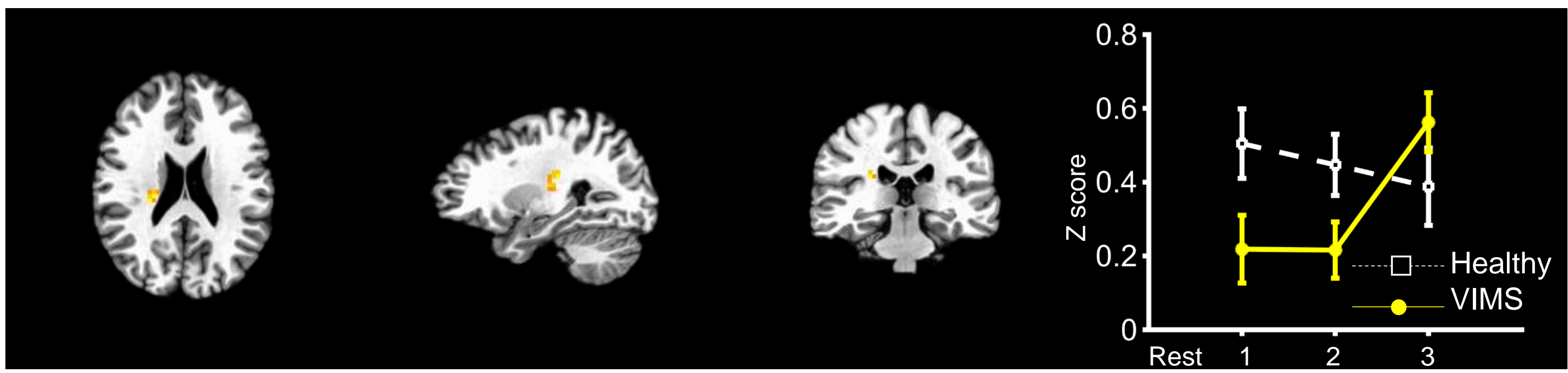

Supplementary Figure 1. Brain regions whose functional connectivity with the seed regions increased in the recovery phase from visually induced motion sickness (VIMS)

The same as Supplementary Figure 1a but for right insula. 


\section{L Medial Frontal Gyrus (seed: L Superior Frontal Gyrus)}

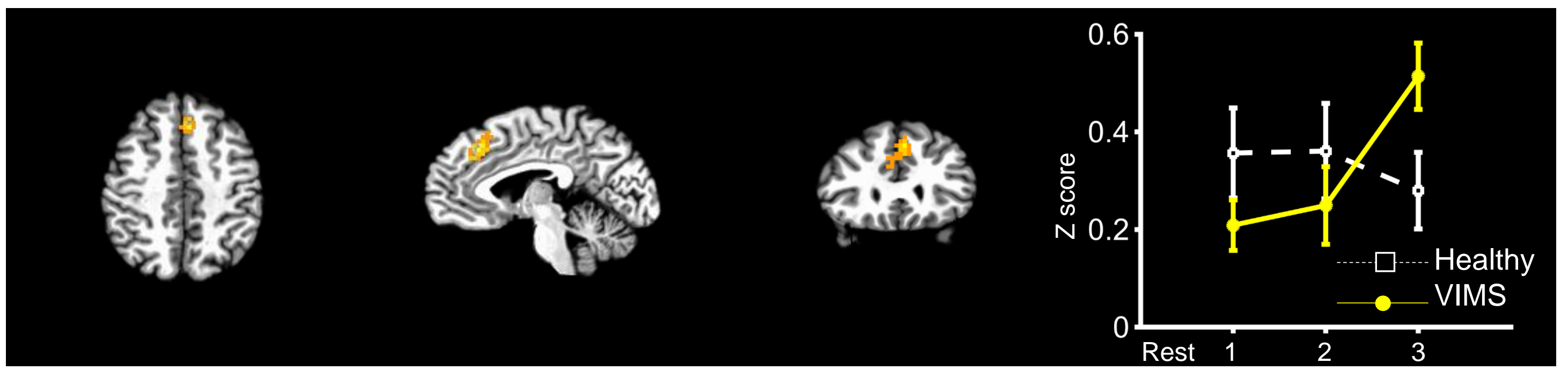

Supplementary Figure 1. Brain regions whose functional connectivity with the seed regions increased in the recovery phase from visually induced motion sickness (VIMS)

The same as Supplementary Figure 1a but for left medial frontal gyrus. 
m 17. L Middle Temporal Gyrus (seed: L Superior Frontal Gyrus)

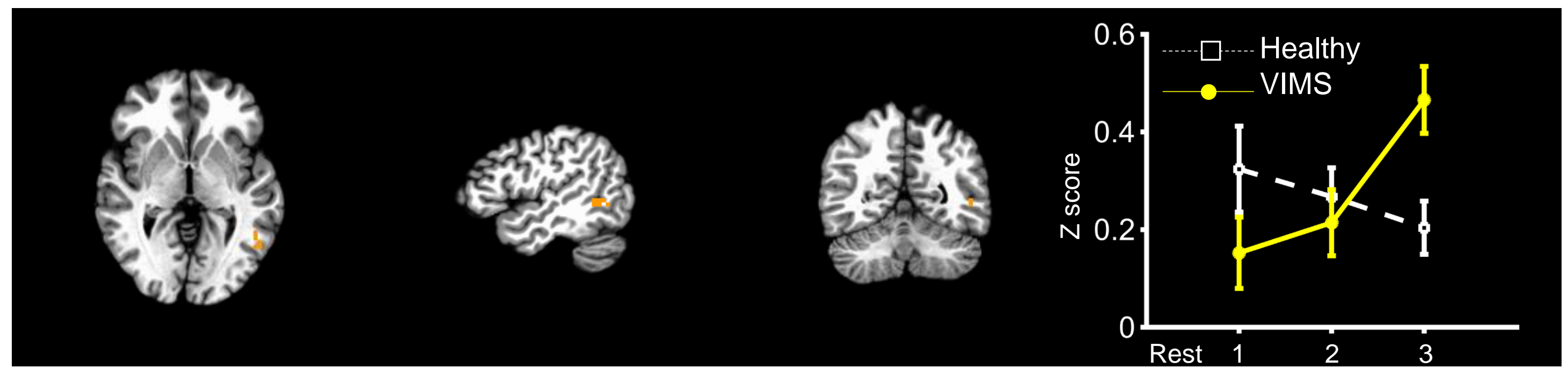

Supplementary Figure 1. Brain regions whose functional connectivity with the seed regions increased in the recovery phase from visually induced motion sickness (VIMS)

The same as Supplementary Figure 1a but for left middle temporal gyrus. 


\section{n 19. L Inferior Frontal Gyrus (seed: R Inferior Frontal Gyrus)}

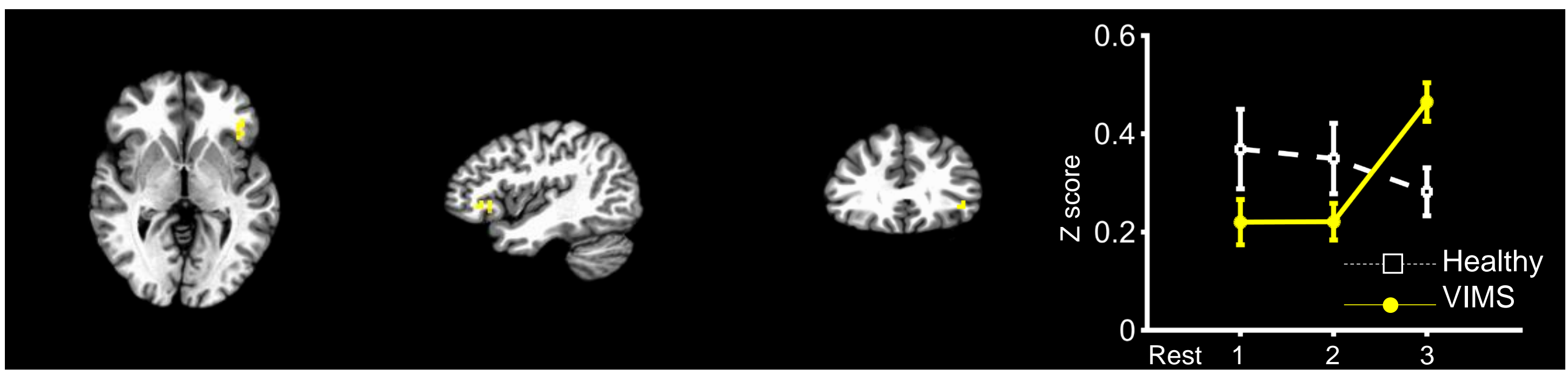

Supplementary Figure 1. Brain regions whose functional connectivity with the seed regions increased in the recovery phase from visually induced motion sickness (VIMS)

The same as Supplementary Figure 1a but for left inferior frontal gyrus. 
a

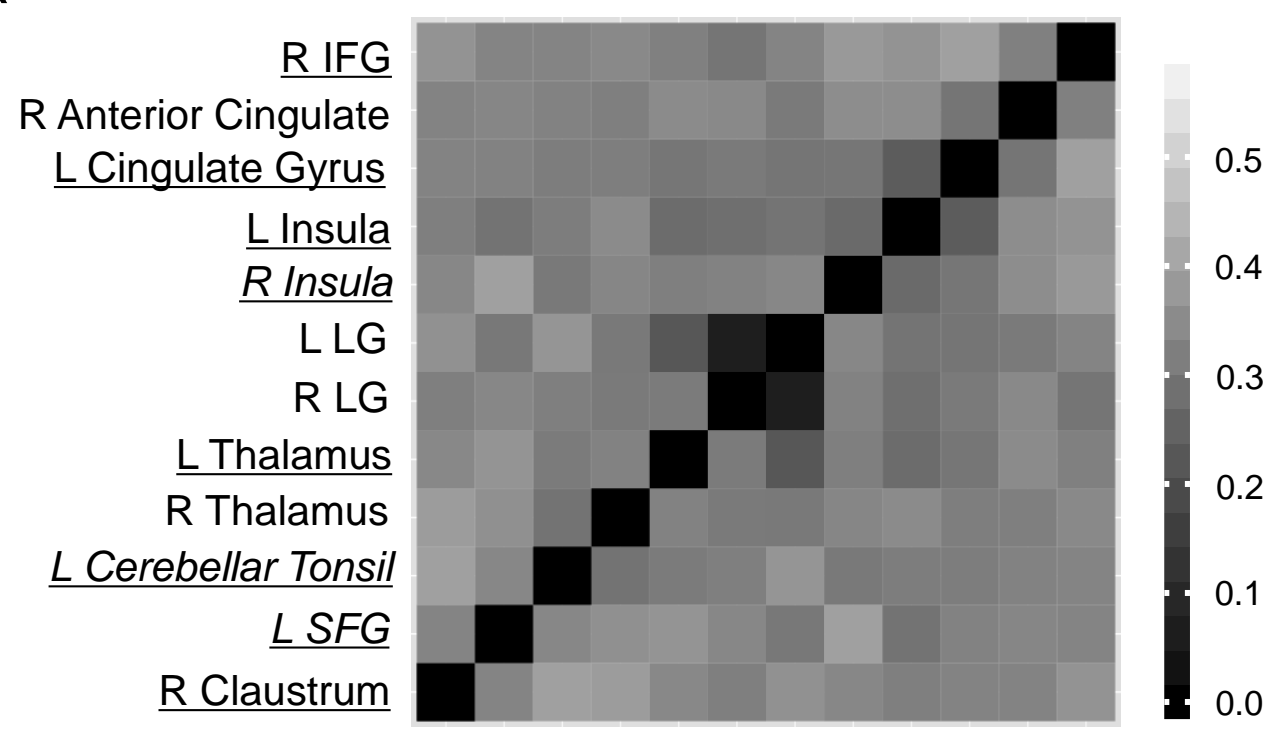

C

VIMS control (average of Rest-1 \& -2)phase

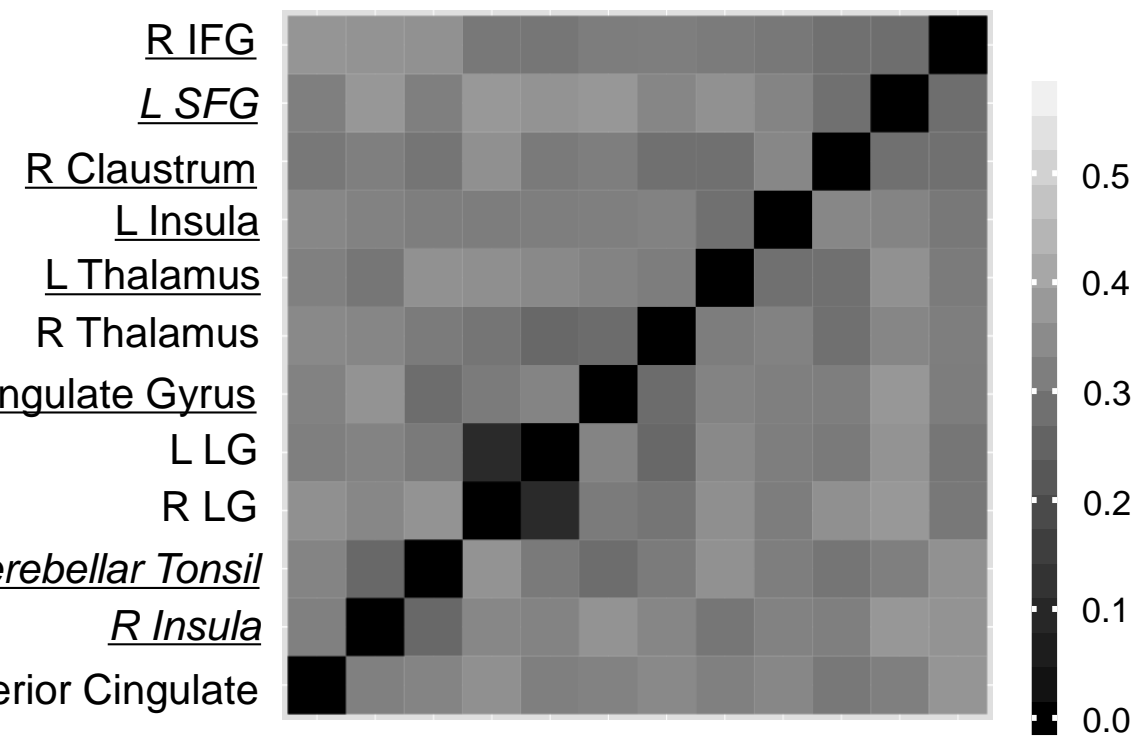

b $^{\text {oto-u.uac.jp }}$

healthy control (Rest-3)

R Anterior Cingulate L LG $R L G$ $L S F G$

$\underline{\mathrm{R} \text { Claustrum }}$ $\underline{L}$ Cerebellar Tonsil

$R$ Thalamus R IFG

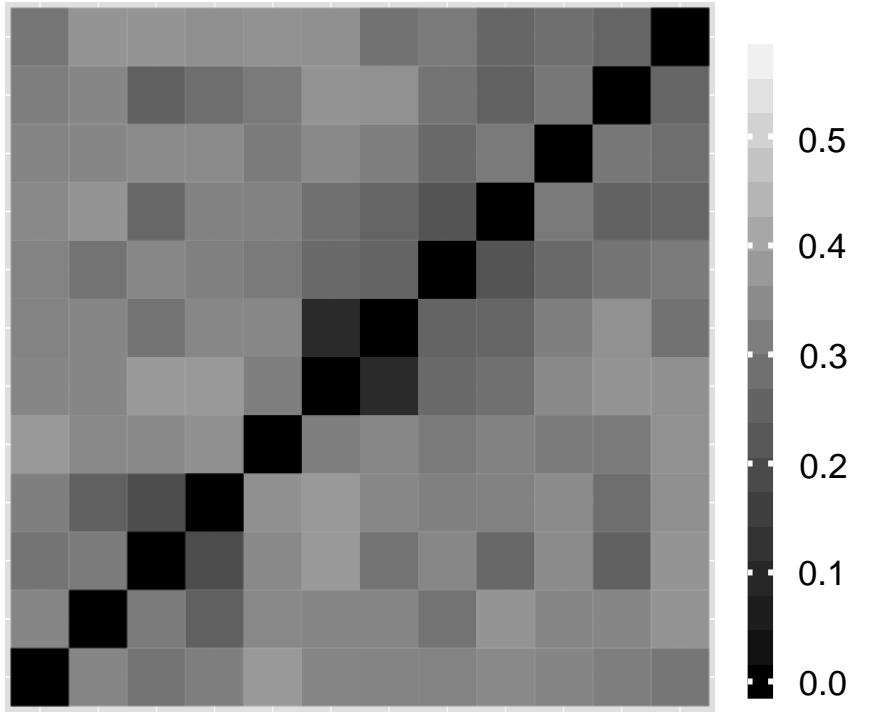

d

healthy control (average of Rest-1 \& -2) phase

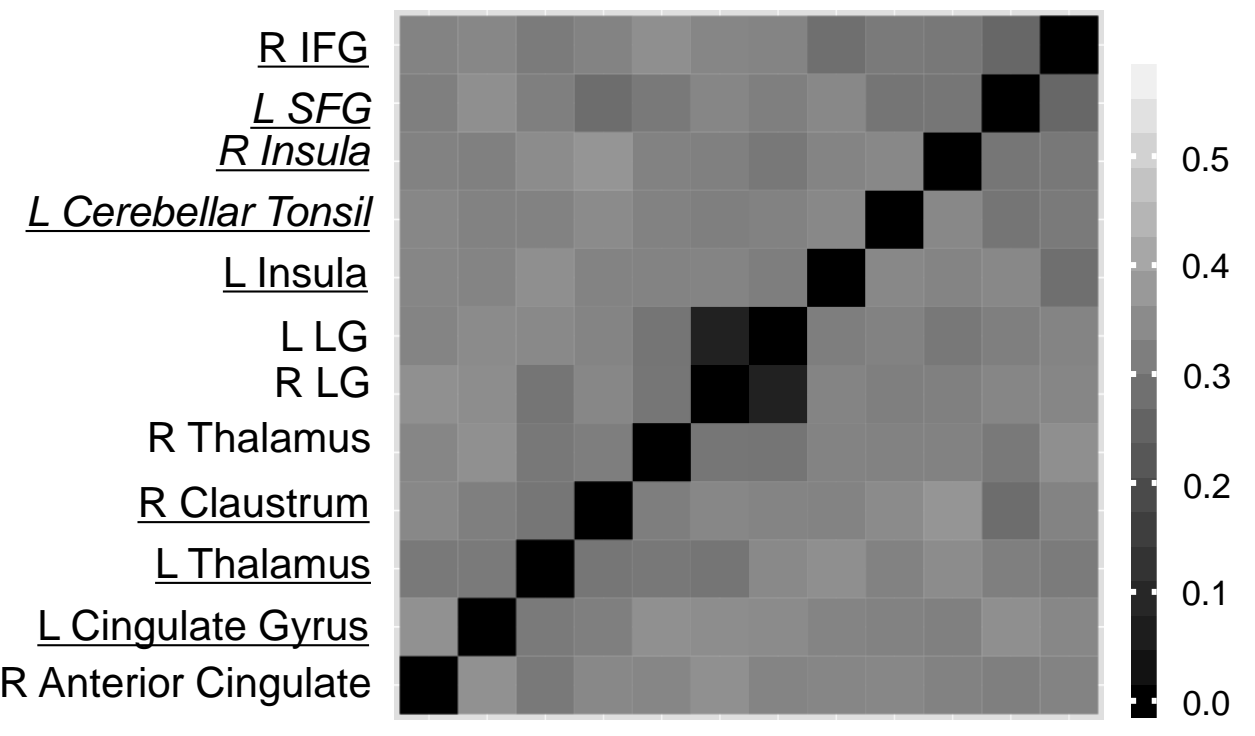
R Anterior Cingulate

Supplementary Figure 2. Distance matrices of the 12 brain regions showing recovery-selective increases in connectedness

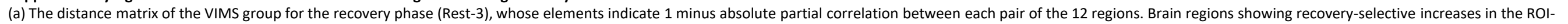

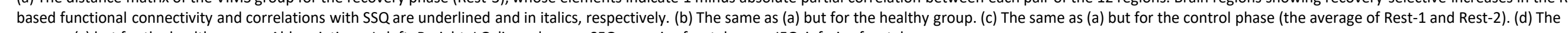
same as (c) but for the healthy group. Abbreviations: L, left; R, right; LG, lingual gyrus; SFG, superior frontal gyrus; IFG, inferior frontal gyrus. 
